\title{
Identification and characterization of differentially expressed genes in Caenorhabditis elegans in response to pathogenic and nonpathogenic Stenotrophomonas maltophilia
}

Leah J. Radeke and Michael A. Herman ${ }^{*}$

\begin{abstract}
Background: Stenotrophomonas maltophilia is an emerging nosocomial pathogen that causes infection in immunocompromised patients. S. maltophilia isolates are genetically diverse, contain diverse virulence factors, and are variably pathogenic within several host species. Members of the Stenotrophomonas genus are part of the native microbiome of C. elegans, being found in greater relative abundance within the worm than its environment, suggesting that these bacteria accumulate within C. elegans. Thus, study of the C. elegans-Stenotrophomonas interaction is of both medical and ecological significance. To identify host defense mechanisms, we analyzed the $C$. elegans transcriptomic response to S. maltophilia strains of varying pathogenicity: K279a, an avirulent clinical isolate, JCMS, a virulent strain isolated in association with soil nematodes near Manhattan, KS, and JV3, an even more virulent environmental isolate.

Results: Overall, we found 145 genes that are commonly differentially expressed in response to pathogenic $S$. maltophilia strains, $89 \%$ of which are upregulated, with many even further upregulated in response to JV3 as compared to JCMS. There are many more JV3-specific differentially expressed genes (225, $11 \%$ upregulated) than JCMS-specific differentially expressed genes (14,86\% upregulated), suggesting JV3 has unique pathogenic mechanisms that could explain its increased virulence. We used connectivity within a gene network model to choose pathogen-specific and strain-specific differentially expressed candidate genes for functional analysis. Mutations in 13 of 22 candidate genes caused significant differences in C. elegans survival in response to at least one S. maltophilia strain, although not always the strain that induced differential expression, suggesting a dynamic response to varying levels of pathogenicity.

(Continued on next page)
\end{abstract}

\footnotetext{
* Correspondence: mherman5@unl.edu

School of Biological Sciences, University of Nebraska-Lincoln, Lincoln, NE 68588, USA

(c) The Author(s). 2020 Open Access This article is licensed under a Creative Commons Attribution 4.0 International License, which permits use, sharing, adaptation, distribution and reproduction in any medium or format, as long as you give appropriate credit to the original author(s) and the source, provide a link to the Creative Commons licence, and indicate if changes were made. The images or other third party material in this article are included in the article's Creative Commons licence, unless indicated otherwise in a credit line to the material. If material is not included in the article's Creative Commons licence and your intended use is not permitted by statutory regulation or exceeds the permitted use, you will need to obtain permission directly from the copyright holder. To view a copy of this licence, visit http://creativecommons.org/licenses/by/4.0/. The Creative Commons Public Domain Dedication waiver (http://creativecommons.org/publicdomain/zero/1.0/) applies to the data made available in this article, unless otherwise stated in a credit line to the data.
} 
(Continued from previous page)

Conclusions: Variation in observed pathogenicity and differences in host transcriptional responses to S. maltophilia strains reveal that strain-specific mechanisms play important roles in S. maltophilia pathogenesis. Furthermore, utilizing bacteria closely related to strains found in C. elegans natural environment provides a more realistic interaction for understanding host-pathogen response.

Keywords: Caenorhabditis elegans, Stenotrophomonas maltophilia, Differential expression, Innate immune response, Bacteria

\section{Background}

Stenotrophomonas maltophilia is a Gram-negative, nosocomial pathogen that can cause infection in immunocompromised patients. S. maltophilia is often found in patients with cystic fibrosis and lung cancer, and is associated with infections such as pneumonia, endocarditis, bacteremia, and meningitis [1]. Although not highly virulent, S. maltophilia is multi-drug resistant and capable of forming biofilms [2,3] thus developing treatment methods for this pathogen is becoming an increasing concern. S. maltophilia is ubiquitous within the environment, commonly found in aqueous sources, soils, and associated with plant roots, and can also be isolated in hospitals from water sources and medical devices $[1,4]$.

Sequencing and functional analyses have identified both similarities and differences in virulence factors such as antibiotic resistance and quorum sensing mechanisms in clinical and environmental isolates of S. maltophilia [5-10]. Therefore, strain diversity appears to result in different virulence mechanisms and pathogenic potential $[5,11]$. Although studies have identified virulence factors within $S$. maltophilia genomes, phenotypic analysis using host species is rarely performed, and mechanisms of host responses are poorly understood. Therefore, we recently established Caenorhabditis elegans as a model to study host responses to S. maltophilia infection [12].

C. elegans is an excellent genetic model organism for studying many biological processes, including development, neurobiology, and innate immunity. C. elegans are bacterivores and can be found in the natural environment in decaying fruits and stems where they are in contact with many bacterial species. Recent studies have found that Stenotrophomonas is one of the most abundant genera of bacteria found in the native microbiome of C. elegans [13-15]. Furthermore, Stenotrophomonas is found in higher abundance within the microbiome than in rotting substrates where C. elegans are found [14, 15], suggesting that it colonizes and accumulates in the intestine, a common signature of pathogenesis in C. elegans $[16,17]$. In fact, many of these Stenotrophomonas isolates were found to be detrimental to the health of $C$. elegans, while few were found to be beneficial [14, 18]. This is consistent with previous observations that $S$. maltophilia strains show varying pathogenicity to $C$. elegans, amoeba (Dictyostelium discoideum and Acanthamoeba castellanii), and zebrafish [6, 8, 12]. This suggests that $S$. maltophilia strains utilize different virulence mechanisms that result in different host responses.

Many innate immune pathways in C. elegans are conserved from invertebrates to mammals, making it an excellent model for studying pathogen-host interactions and innate immunity. Briefly, the p38 mitogen-activated protein kinase (MAPK) pathway plays a role in defense against several pathogens, including S. maltophilia, Pseudomonas aeruginosa, Staphylococcus aureus, and Salmonella enterica [12, 19-21]. In addition, activation of the insulin-like signaling pathway decreases bacterial packing, suggesting that regulation of genes by the downstream transcription factor DAF-16 defends against accumulation of bacteria in the intestine [16]. Analyses of mutations affecting genes in these pathways have identified downstream proteins involved in pathogen defense, such as lysozymes, C-lectins, and CUB-domain containing proteins [22, 23].

Although many important innate immune pathways and effectors have been identified, there are differences in responses to different bacterial pathogens. For example, one study comparing responses to intestinal pathogens Serratia marcescens, Enterococcus faecalis, and Photorhabdus luminescens found that only $11 \%$ of genes in $C$. elegans where commonly differentially expressed in response to all three species [24]. This phenomenon could be due to species-specific responses to different pathogens, or the ability of bacteria to manipulate different host responses. Therefore, it is essential to study a variety of pathogens in order to fully understand the complexity of genetic mechanisms underlying pathogen defense. Finally, studying bacteria that have been identified as part of the microbiome of $C$. elegans, or their close relatives, more closely approximates natural interactions and therefore a more realistic response to the bacteria.

We previously used a microarray approach to identify gene expression patterns in response to Escherichia coli OP50 and S. maltophilia strains K279a and JCMS [25]. We found that the genetic response is more specific to the type of $C$. elegans-bacterial interaction rather than 
bacteria species or strain. Here, we further explore these responses using RNA sequencing to identify and characterize the genetic responses of C. elegans to several different $S$. maltophilia strains of varying levels of pathogenicity. Specifically, we performed transcriptomic analysis on C. elegans following exposure to either E. coli OP50 or one of three S. maltophilia strains: two pathogenic environmental isolates, JCMS and JV3, and one nonpathogenic clinical isolate, K279a. Using this experimental set-up, we discovered responses that are common to both pathogenic $S$. maltophilia strains and responses that are strain-specific based on the level of virulence of each strain. We also identified candidate genes involved in both the common and strain-specific responses and determined that several candidate genes were important for survival of $C$. elegans upon exposure to S. maltophilia.

\section{Results}

We used survival as an indicator of bacterial pathogenicity to C. elegans. Survival analyses showed that S. maltophilia strains K279a, JCMS, and JV3, display differing levels of pathogenicity to C. elegans. S. maltophilia K279a, a clinical isolate of S. maltophilia, is not pathogenic, as worms fed K279a have similar bacterial load and survival as do worms fed the standard lab food $E$. coli OP50 [12] (Fig. 1). However, S. maltophilia JCMS, a strain isolated in association with soil nematodes and $S$. maltophilia JV3, another environmental isolate closely related to JCMS, are both detrimental to the survival of C. elegans (Fig. 1). We used the Cox proportional hazards test to quantify these differences by calculating the hazard, or the probability of a nematode dying at a given

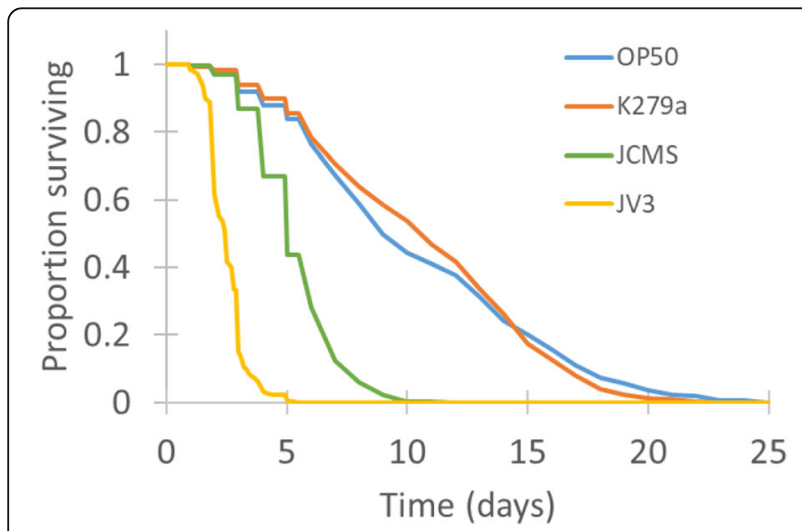

Fig. 1 S. maltophilia strains show varying pathogenicity to C. elegans. Survivorship of wild-type nematodes on S. maltophilia JCMS, K279a, JV3, and E. coli OP50. Survival estimates were determined using Kaplan-Meier estimates generated in R. This data contains all wild-type data collected from experiments in this study, representing 23 individual experiments and $n=516-615$ for each bacterial treatment. Sample sizes, hazard ratios and $p$-values generated form Cox proportional hazards tests are shown in Table 3 time, for each bacterial treatment. Hazards ratios are used to compare relative hazards of different conditions, in this case bacteria, where ratios greater than one indicate treatments that are more detrimental, or hazardous, to the health of C. elegans; whereas hazard ratios less than one indicate more beneficial conditions. C. elegans exposed to JCMS have a hazard of $6.66( \pm 0.07)$, meaning they are 6.66 times more likely to die than C. elegans exposed to OP50, whereas C. elegans exposed to JV3 are 95.64 $( \pm 0.08)$ times more likely to die than $C$. elegans fed OP50. We performed a transcriptomic analysis to discover the genes underlying the response of $C$. elegans to strains of $S$. maltophilia of varying pathogenicity to provide a more comprehensive understanding of C. elegans-pathogen interactions.

\section{C. elegans exhibit common and strain-specific responses to S. maltophilia}

To investigate transcriptomic responses to S. maltophilia, RNA-sequencing was performed after $12 \mathrm{~h}$ of exposure to pathogenic S. maltophilia JCMS or JV3, or nonpathogenic S. maltophilia K279a or E. coli OP50. The 12-h time point was chosen based on previous observations that accumulation of bacteria occurs by this time [12] but S. maltophilia JV3-induced mortality has not yet begun (Fig. 1). In addition, other groups have identified transcriptional changes at $4-8 \mathrm{~h}$ of exposure to pathogens, including S. aureus, Bacillus thuringiensis, and $P$. aeruginosa $[23,26,27]$. Therefore, at $12 \mathrm{~h}$, pathogen recognition has begun, but transcriptional changes associated with aging and mortality, which correlate with a decreased immune response [28], should not complicate interpretation of data.

Overall gene expression patterns were analyzed using a heatmap of genes that were significantly differentially expressed between any two treatments (Fig. 2; Supplemental Table 1, Additional File 1). Transcripts were considered differentially expressed if they had a false discovery rate (FDR)-adjusted $p$-value of less than 0.05 and an absolute fold change greater than two. Gene expression profiles showed clustering of nonpathogenic (K279a and E. coli OP50) and pathogenic (JCMS and JV3) treatments (Fig. 2). Although there are differences between expression profiles of the nonpathogenic strains, suggesting the existence of a species-specific response, the expression profiles of the nonpathogenic treatments were more similar than that of the pathogenic treatments (Fig. 2). Therefore, to identify the common response to pathogenic $S$. maltophilia, we compared differentially expressed genes in C. elegans between pathogenic and nonpathogenic treatments (Fig. 3). In total, 1296 genes were significantly differentially expressed when comparing worms fed any pathogenic (JV3 and JCMS) to any nonpathogenic (K279a and E. 


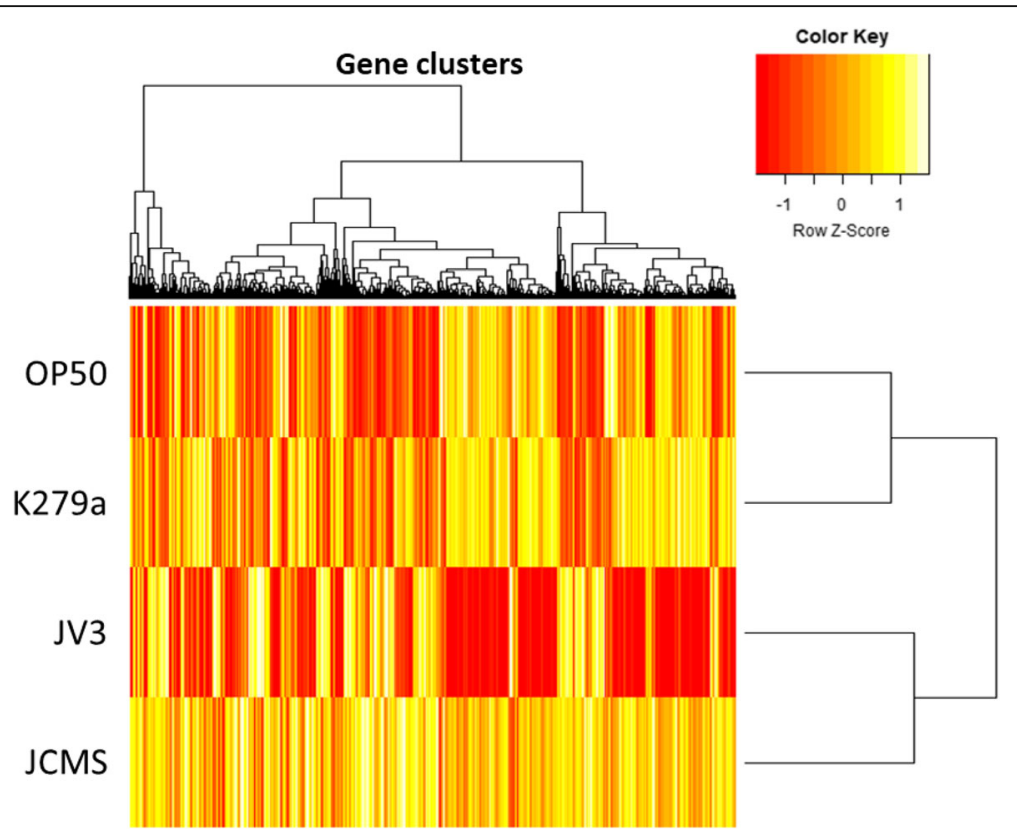

Fig. 2 Heatmap of C. elegans differentially expressed genes in response to S. maltophilia. Differentially expressed genes from RNA-sequencing include genes with fold-change $>2$ and FDR-adjusted $p$-value $<0.05$ between any treatment comparisons. Heatmap was generated with log transformed FPKM values and visualized using the heatmap.2 function in gplots package in R. Dendrogram on the y-axis represents degree of similarity of treatments based on gene expression profiles. Dendrogram on the $x$-axis represents degree of similarity of gene clusters based on expression profile across treatments. Gene expression is color coded, with red indicating lower expression and yellow indicating higher expression. The scaling option was used so that each gene is individually normalized across treatments

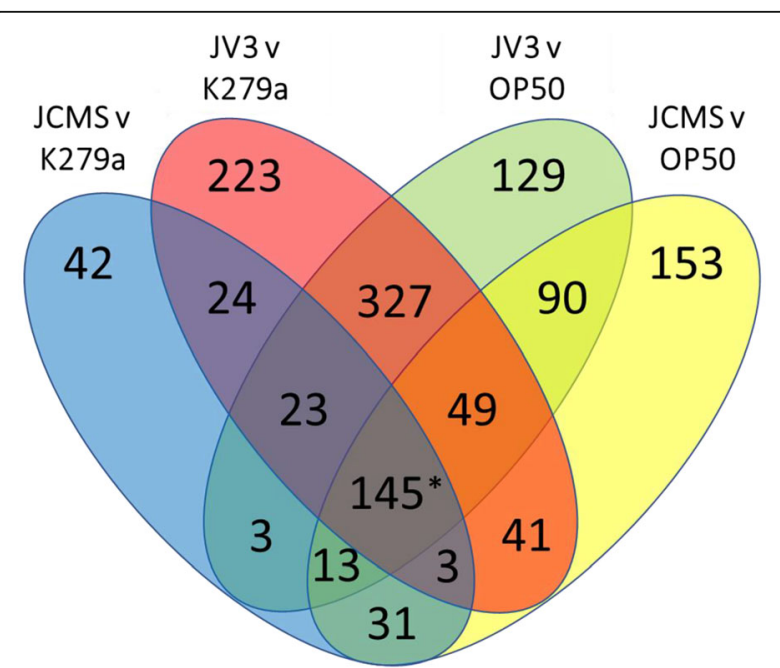

Fig. 3 C. elegans expresses a common set of 145 genes in response to pathogenic S. maltophilia strains. Differential expression was determined between each pathogenic and nonpathogenic comparison, with the number of significantly differentially expressed genes indicated between each set of comparisons. Genes included are differentially expressed between the specified treatments with fold-change $>2$ and FDR-adjusted $p$-value $<0.05$. 145 genes were commonly differentially expressed between all pathogenic (JCMS and JV3) and nonpathogenic (K279a and OP50) treatments, or the common pathogenic S. maltophilia response (CPSR), indicated by the asterisk coli OP50) strain, with $11 \%$ (145) commonly differentially expressed between all pathogenic and nonpathogenic comparisons (Fig. 3, Supplemental Table 2; Supplemental Table 3, Additional File 1) These most likely represent a core set of genes that are regulated upon exposure to pathogenic S. maltophilia and are therefore referred to as the "common pathogenic S. maltophilia response" (CPSR). Because these genes are differentially expressed in response to pathogenic vs nonpathogenic strains of the same species, this should remove general responses to $S$. maltophilia and represent genes specifically involved in pathogen response to S. maltophilia. Of the 145 CPSR genes, 129 (89\%) were upregulated in response to the pathogenic strains as compared to the nonpathogenic strains, whereas 15 (10\%) were downregulated (Supplemental Table 3, Additional File 1). One gene, lys-10, is upregulated in response to the pathogenic strains compared to OP50 but downregulated in response to pathogenic strains compared to K279a. Interestingly, most upregulated genes, 90 of 129, were even further upregulated in response to JV3 as compared to JCMS. Because JV3 is more virulent than JCMS, this suggests that the level of virulence influences the expression of S. maltophilia-induced genes.

A gene ontology (GO) enrichment analysis was performed on all CPSR genes using the Database for Annotation, Visualization and Integrated Discovery (DAVID) 
$[29,30]$ to identify common cellular components, biological processes, and molecular functions of these genes. The terms "biological process of innate immune response" $(\mathrm{FDR}=1.48 \mathrm{E}-51)$, "biological process of defense to Gram-negative bacterium" (FDR $=4.19 \mathrm{E}-11)$, "molecular function of carbohydrate binding" (FDR = $1.84 \mathrm{E}-4)$, and "cellular component of membrane raft" $(\mathrm{FDR}=8.13 \mathrm{E}-20)$ were all significantly enriched among the CPSR genes (Table 1). We also analyzed GO enrichment for the up- and down-regulated genes separately. Enriched GO terms for the upregulated CPSR genes were very similar to those for all CPSR genes (Supplemental Table 6, Additional File 2), whereas analysis of downregulated genes resulted in no significant GO terms with $\mathrm{FDR}<0.05$, possibly due to the small number of downregulated CPSR genes (15).

To identify JV3- and JCMS-specific responses, we identified genes that were differentially expressed in response to JV3 and JCMS as compared to all other strains. We found 31 genes differentially expressed in response to JCMS vs the nonpathogenic strains and 327 genes differentially expressed in response to JV3 vs the nonpathogenic strains (Fig. 3). We found that 14 of the 31 JCMS vs nonpathogenic strains genes were also differentially expressed between JV3 and JCMS (Supplemental Table 4, Additional File 1). These genes are specifically regulated upon exposure to $S$. maltophilia JCMS and are therefore referred to as the "JCMS-specific response" (JSR). Of the 14 JSR genes, 12 are upregulated in response to JCMS as compared to all other strains, whereas two are downregulated (Supplemental Table 4, Additional File 1).

We found that 225 of the 327 JV3 vs nonpathogenic strain genes were also differentially expressed between JV3 and JCMS (Fig. 3; Supplemental Table 5, Additional File 1). These genes are specifically regulated upon exposure to S. maltophilia JV3 and are referred to as the
"JV3-specific response" (VSR). Although most CPSR genes are upregulated in response to JV3, a majority $(89 \%)$ of the VSR genes are downregulated in response to JV3 as compared to the other strains (Supplemental Table 5, Additional File 1). This suggests that one virulence mechanism employed by JV3 may be to reduce expression of a variety of host genes. GO enrichment analyses of these genes reveals enrichment of several metabolic processes and enzymes, including "biological process of flavonoid glucuronidation" (FDR $=3.02 \mathrm{E}-09$ ), "biological process of oxidation-reduction process" (FDR = 0.0433), "molecular function of glucuronosyltransferase activity" (FDR =9.7E-06), and "molecular function of carboxylic ester hydrolase activity" (FDR = 7.24E-04) (Table 2).

Again, we also analyzed GO enrichment for the upand down-regulated VSR genes separately. Downregulated enriched GO terms were very similar to those for all VSR genes (Supplemental Table 7, Additional File 2), whereas analysis of upregulated genes resulted in no significant $G O$ terms with and FDR $<0.05$, possibly due to the small number of upregulated VSR genes.

\section{Gene network analysis to prioritize important response genes}

We next wanted to determine whether the CPSR, JSR, and VSR genes are important for the response to both pathogenic S. maltophilia strains (CPSR genes) or to specific strains of S. maltophilia (JSR and VSR genes). To do this, we utilized WormNet, a probabilistic gene network model, to prioritize genes for functional analysis [31]. WormNet uses both direct physical and/or genetic interactions as well as inferred interactions to create a gene network that comprises $75.4 \%$ (15,139 genes) of the C. elegans genome, resulting in 999,367 functional linkages [31]. Previously, gene networks have been used to identify genes essential for $C$. elegans development and

Table 1 Innate immune response GO terms significantly enriched in common pathogenic S. maltophilia response (CPSR) genes

\begin{tabular}{|c|c|c|c|c|}
\hline GO category & Term & Count & $\%$ & FDR \\
\hline \multirow[t]{6}{*}{ Biological process } & response to stimulus & 60 & 43.48 & 4.19E-11 \\
\hline & response to stress & 55 & 39.86 & 3.47E-31 \\
\hline & defense response & 52 & 37.68 & $2.33 \mathrm{E}-46$ \\
\hline & innate immune response & 50 & 36.23 & $1.48 \mathrm{E}-51$ \\
\hline & defense response to bacterium & 16 & 11.59 & $6.58 \mathrm{E}-12$ \\
\hline & defense response to Gram-negative bacterium & 14 & 10.14 & 4.19E-11 \\
\hline Molecular function & carbohydrate binding & 12 & 8.70 & $1.84 \mathrm{E}-04$ \\
\hline Cellular component & membrane raft & 17 & 12.32 & $8.13 E-20$ \\
\hline
\end{tabular}

Gene ontology (GO) enrichment analysis was performed on the CPSR genes using DAVID Bioinformatics Resources 6.8 . Of the 145 CPSR genes, 138 were identified in DAVID and considered for analysis. GO analysis identifies terms relating to the biological process, molecular function, or cellular component that are significantly enriched among a list of genes. Indented terms indicate child terms, or subcategories, of the term listed above, with the parent term left-aligned. Note that the degree of indention of each term does not reflect absolute GO term level within each category. Count is the number of genes corresponding to each GO term. Percent is the count/138 total analyzed. FDR is the false discovery rate-corrected EASE enrichment score to account for multiple testing. Only terms with FDR $<0.05$ and the most descriptive term for each unique gene list are shown 
Table 2 Metabolism and enzyme GO terms significantly enriched in S. maltophilia JV3-specific response (VSR) genes

\begin{tabular}{|c|c|c|c|c|}
\hline GO category & Term & Count & $\%$ & FDR \\
\hline \multirow[t]{9}{*}{ Biological process } & single-organism metabolic process & 49 & 22.68 & $2.47 \mathrm{E}-06$ \\
\hline & Small molecule metabolic process & 27 & 12.5 & 5.27E-04 \\
\hline & organic acid metabolic process & 22 & 10.19 & $1.64 \mathrm{E}-05$ \\
\hline & carboxylic acid metabolic process & 21 & 9.72 & 2.21E-05 \\
\hline & monocarboxylic acid metabolic process & 18 & 8.33 & 8.53E-07 \\
\hline & flavonoid glucuronidation & 14 & 6.48 & $3.02 \mathrm{E}-09$ \\
\hline & flavonoid biosynthetic process & 14 & 6.48 & $3.02 \mathrm{E}-09$ \\
\hline & oxidation-reduction process & 21 & 9.72 & 0.0433 \\
\hline & transition metal ion transport & 7 & 3.24 & 0.0468 \\
\hline \multirow[t]{6}{*}{ Molecular function } & catalytic activity & 89 & 41.20 & 0.00174 \\
\hline & transferase activity, transferring glycosyl groups & 17 & 7.87 & 0.00203 \\
\hline & transferase activity, transferring hexosyl groups & 14 & 6.48 & $1.76 \mathrm{E}-07$ \\
\hline & glucuronosyltransferase activity & 12 & 5.56 & $9.70 \mathrm{E}-06$ \\
\hline & UDP-glycosyltransferase activity & 16 & 7.41 & 0.00155 \\
\hline & carboxylic ester hydrolase activity & 11 & 5.09 & 7.24E-04 \\
\hline Cellular component & extracellular region & 20 & 9.26 & 0.0295 \\
\hline
\end{tabular}

Gene ontology (GO) enrichment analysis was performed on the VSR genes using DAVID Bioinformatics Resources 6.8. Of the 225 VSR genes, 216 were identified in DAVID and considered for analysis. GO analysis identifies terms relating to the biological process, molecular function, or cellular component that are significantly enriched among a list of genes. Indented terms indicate child terms, or subcategories, of the term listed above, with the parent term left-aligned. Note that the degree of indention of each term does not reflect absolute GO term level within each category. Count is the number of genes corresponding to each GO term. Percent is the count/216 total analyzed. FDR is the false discovery rate-corrected EASE enrichment score to account for multiple testing. Only terms with FDR < 0.05 and the most descriptive term for each unique gene list are shown

survival under standard conditions, as well as identification of genes associated with particular diseases [32, 33]. In addition, we previously found this method to be helpful to identify functionally important $S$. maltophilia-induced genes [25]. Therefore, we hypothesize that the most connected genes within the gene network play a significant role in S. maltophilia response and are therefore better candidates for functional analyses. In addition to gene network connectivity, we preferentially chose genes for functional analysis with available alleles, either from the Caenorhabditis Genetics Center (CGC) or previously generated in our lab, and generated mutant alleles using CRISPR/Cas9 for additional genes that were expressed at higher levels.

Of the 145 CPSR genes, 73 were connected within the gene network with an AUC of $0.6972(p=1.8 \mathrm{E}-16)$ (Fig. 4; Supplemental Table 8, Additional File 3). The AUC is the area under the receiver operating characteristic (ROC) curve and provides a measure for the recovery of true-positive genes as compared to false-positive genes [31]. A random network would have an AUC of 0.5 , whereas a network representing perfect prediction of all connections would have an AUC of one; therefore, an AUC of $0.6972(p=1.81 \mathrm{E}-16)$ suggests relatively high predictive power of gene connections. Each connected gene is ranked based on the number of connections as well as the strength of the evidence for those connections [32], with some of the highest-ranking CPSR genes including lys-1, lys-2, dod-22, dod-19, and clec67 (Supplemental Table 8, Additional File 3). Previous studies have identified these genes as downstream effectors of defense pathways or directly involved in response to bacterial pathogen challenge $[12,22,34$, 35]. Mutant alleles were available for these genes, along with alleles of several other genes highly connected within the network, including F55G11.8, $Z K 6.11, T 24 B 8.5$, and $s c l-2$. In addition, we had previously generated mutant alleles affecting K08D8.4, B0024.4, and F08G2.5 using CRISPR/Cas9 (Additional File 4).

Of the 225 VSR genes, 128 are connected within the network (AUC $=0.6776, p=2.81 \mathrm{E}-22$ ) (Fig. 5; Supplemental Table 9, Additional File 3). Available alleles of several of the highest-ranking genes in this network, including sodh-1, dhs-3, F13D12.6, pho-1, acdh-1, C55A6.7, dhs-2, and F08A8.4 were used for functional analysis.

Because of the small number of JSR genes, WormNet was not used to prioritize these genes for functional analysis. Many of these genes had very low overall expression. Therefore, genes were chosen if the total fragments per kilobase per million mapped reads (FPKM) for all four treatments was greater than 30 . We used an available allele of the upregulated gene $n h r-110$ and used CRISPR/Cas9 to generate a deletion of the downregulated gene WO2A2.8 for functional analysis (Additional File 4). 


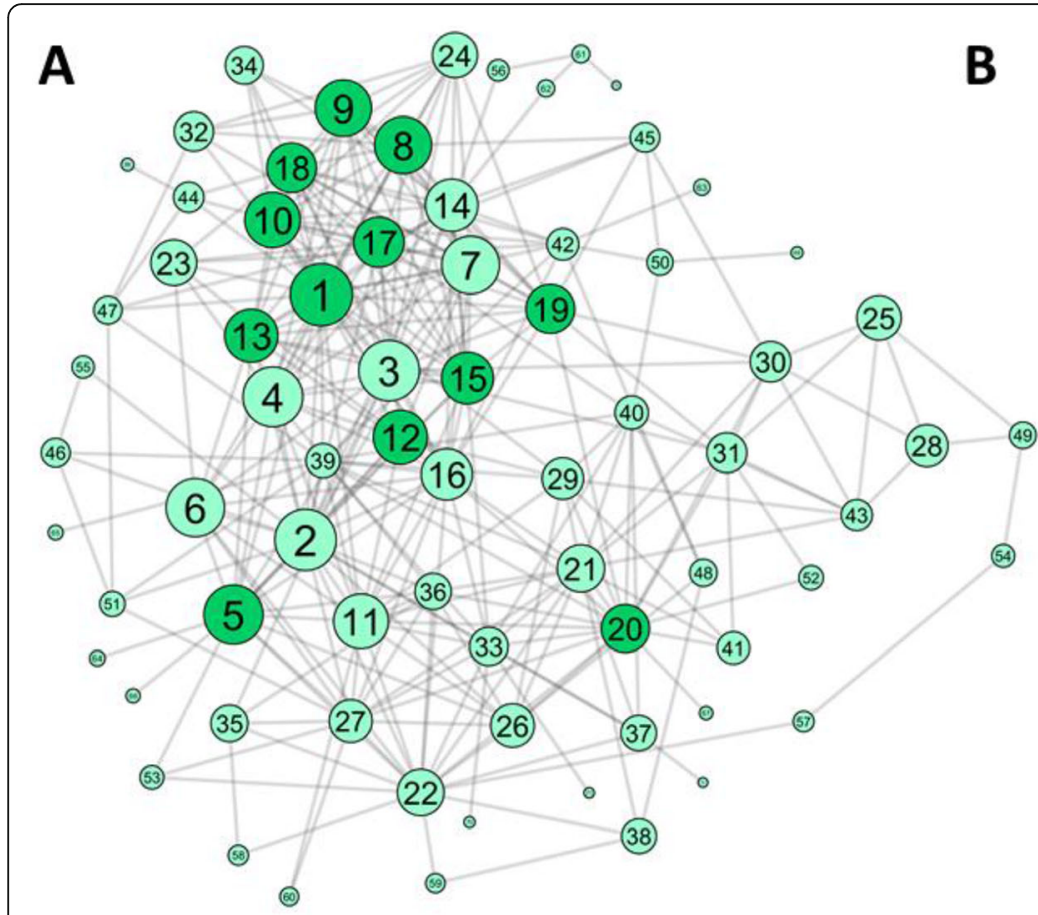

$\begin{array}{clrr}\text { Rank } & \text { Gene } & \text { Score } & \text { Regulation } \\ 1 & \text { lys-2 } & 3.67 & \text { up } \\ 2 & \text { C49G7.10 } & 3.65 & \text { up } \\ 3 & \text { F19B2.5 } & 3.52 & \text { up } \\ 4 & \text { T24C4.4 } & 3.36 & \text { up } \\ 5 & \text { FO8G2.5 } & 3.26 & \text { up } \\ 6 & \text { KO9D9.1 } & 3.19 & \text { up } \\ 7 & \text { C17H12.6 } & 3.1 & \text { up } \\ 8 & \text { dod-22 } & 3.09 & \text { up } \\ 9 & \text { F55G11.8 } & 3.08 & \text { up } \\ 10 & \text { ZK6.11 } & 3.06 & \text { up } \\ 11 & \text { CO8E8.4 } & 3.03 & \text { up } \\ 12 & \text { T24B8.5 } & 2.96 & \text { up } \\ 13 & \text { KO8D8.4 } & 2.92 & \text { up } \\ 14 & \text { FO8G5.6 } & 2.89 & \text { up } \\ 15 & \text { BOO24.4 } & 2.85 & \text { up } \\ 16 & \text { cpr-3 } & 2.82 & \text { up } \\ 17 & \text { lys-1 } & 2.81 & \text { up } \\ 18 & \text { dod-19 } & 2.75 & \text { up } \\ 19 & \text { clec- } 67 & 2.73 & \text { up } \\ 20 & \text { scl-2 } & 2.55 & \text { down }\end{array}$

Fig. 4 Gene network analysis was used to prioritize CPSR genes. WormNet v2, a probabilistic functional gene network model, was queried with the 145 genes that were differentially expressed in response to non-pathogenic vs. pathogenic strains. 73 of the 145 genes are connected to one another (AUC = 0.6942, $p=1.8137 \mathrm{e}-16$ ). a Network visualized using Cytoscape 3.5.1. Green circles represent individual genes and grey lines represent known or predicted connections between genes. Genes are numbered and sized based on rank (Supplemental Table 8, Additional File 3); darker green circles indicate genes that were chosen for functional analysis. $\mathbf{b}$ Top 20 ranked genes. Rank is determined based on score, which is calculated based on number of connections and the strength of evidence for those connections. Regulation indicates direction of differential expression in response to pathogenic compared to nonpathogenic strains

Functional analysis of common S. maltophilia and strainspecific genes

Survivorship of mutants compared to wild-type C. elegans, quantified by Cox proportional hazards test, was used to determine whether candidate genes were important for response to treatment bacteria E. coli OP50 and S. maltophilia K279a, JCMS, and JV3. We tested the simple hypothesis that CPSR genes are important for response to both JCMS and JV3, JSR genes are important for response to JCMS, and VSR genes are important for response to JV3; therefore, mutants of these genes will result in increased or decreased susceptibility to JCMS and JV3, just JCMS, or just JV3, respectively, as compared to wild-type.

Mutations in four of the 12 CPSR candidate genes (lys-1, K08D8.4, ZK6.11, and dod-19) caused significantly increased susceptibly to JCMS, while three mutations (B0024.4, K08D8.4, and T24B8.5) also caused increased susceptibility to JV3 (Fig. 6; Table 3; Additional File 5). Mutations in two of these genes (K08D8.4 and lys-1) also increased susceptibility to K279a (Fig. 6; Table 3; Additional File 5). All of these genes, apart from B0024.4, were previously reported to play a role in innate immune response based on GO terms. In addition, mutations in lys-1 caused increased susceptibility to $E$. coli OP50, while $s c l-2$ caused decreased susceptibility to OP50. Overall, mutations in seven of the 12 CPSR genes caused significant differences in survival in response to at least one bacterial treatment.

Mutations in three of the eight VSR candidate genes (acox-1.4, dhs-3, dhs-2) caused significantly decreased survival in response to JV3, while mutations in acdh-1 resulted in increased lifespan (Fig. 6; Table 3; Additional File 6). However, worms with mutations in all four of these genes also result in significant differences in survival in response to at least one other bacterial strain tested, suggesting that although these genes are specifically differentially expressed in response to JV3, they are also important for survival under other conditions. Additionally, mutations in C55A6.7 and pho-1 decreased susceptibility to $\mathrm{K} 279 \mathrm{a}$, and mutations in pho-1 and sodh-1 increased susceptibility to JCMS (Fig. 6; Table 3; Additional File 6). Overall, seven of the eight VSR genes are important for the response to at least one S. maltophilia strain. Interestingly, while only two of the eight genes are involved in innate immune response based on GO terms (C55A6.7 and $a c d h-1)$, seven of the eight genes have GO terms associated with metabolic 


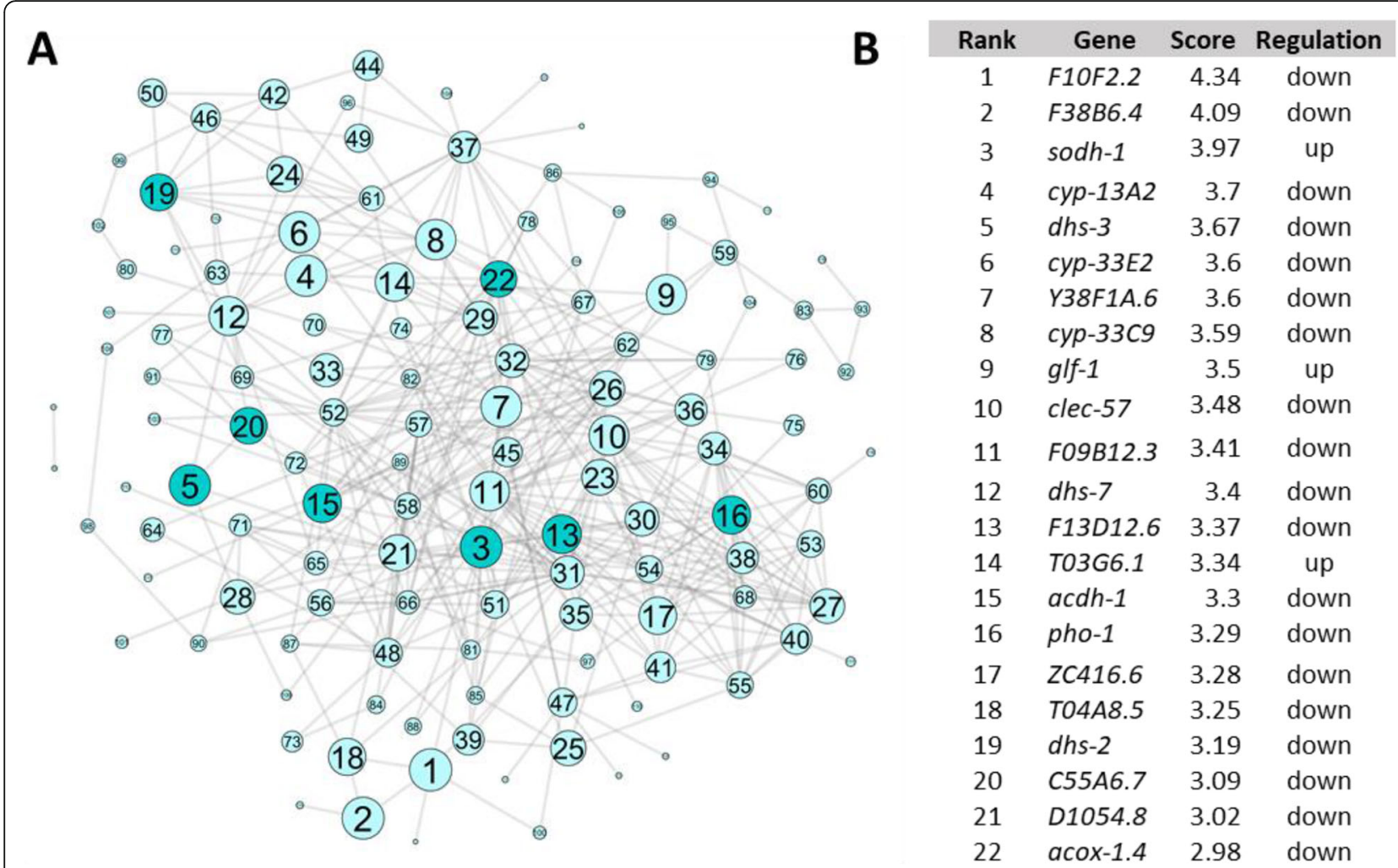

Fig. 5 Gene network analysis was used to prioritize VSR genes. WormNet v2, a probabilistic functional gene network model was queried with the $225 \mathrm{JV} 3$-specific response (VSR) genes. 128 of the 225 genes are connected to one another (AUC=0.6776, $p=2.81 \mathrm{E}-22$ ). a Network was visualized using Cytoscape 3.5.1. Blue circles represent individual genes and grey lines represent known or predicted connections between genes. Genes are numbered and sized based on rank (Supplemental Table 9, Additional File 3); darker blue circles indicate genes that were chosen for functional analysis. b Top 22 ranked genes. Rank is determined based on score, which is calculated based on number of connections and the strength of evidence for those connections. Regulation indicates direction of differential expression in response to JV3 compared to all other strains

processes, including oxidation-reduction (acdh-1, acox1.4, dhs-3, dhs-2, sodh-1), proteolysis (F13D12.6), and dephosphorylation (pho-1). In addition, all of these genes, except $d h s-2$ and $C 55 A 6.7$, have been shown to be expressed in the intestine [36-39] (Fig. 7), the site of S. maltophilia accumulation and proposed pathogenesis [12]. Therefore, although these genes do not seem to be important exclusively for JV3 survival, they do seem to be important for survival in response to $S$. maltophilia overall. Mutations in $a c d h-1$ and acox-1.4 also increased susceptibility to $E$. coli OP50, possibly suggesting a more general role in C. elegans survival.

Only two JSR genes were functionally analyzed, $n h r-$ 110 and W02A2.8. nhr-110 mutants were significantly less susceptible to OP50, but no differences were seen in survival in response to JCMS or other S. maltophilia strains (Fig. 6; Table 3; Additional File 7).

In addition to survival analyses, we visualized the expression patterns for several CPSR and VSR genes. Several transgenic strains were available from stock centers, including transcriptional reporters for T24B8.5, acdh-1, sodh-1, and a translational reporter for dhs-3. We also generated translational reporters for K08D8.4 and F19B2.5. Intensity and location of expression were measured in response to all $S$. maltophilia strains and E. coli OP50 at 12 and $24 \mathrm{~h}$ of exposure. Interestingly, at $12 \mathrm{~h}$, except for $T 24 B 8.5$, expression patterns did not reflect the RNA-sequencing results (Additional File 8). However, at $24 \mathrm{~h}$, expression profiles of all expression constructs across treatments correlated with the RNAsequencing results (Fig. 7; Additional File 8). This delay in observed differential expression of reporter constructs could be due to multiple factors, including folding and processing of fluorescent proteins, potential degradation in down regulated tissues or accumulation of visible amounts of fusion proteins. However, overall, these observed patterns validate our transcriptomic results.

All of these genes were expressed in the intestine, but localization of expression was also seen in the hypodermis (sodh-1 and acdh-1), muscle (sodh-1), nervous system (sodh-1), and head (K08D8.4, sodh-1, and acdh-1) (Additional File 9). The intestine and hypodermis are 


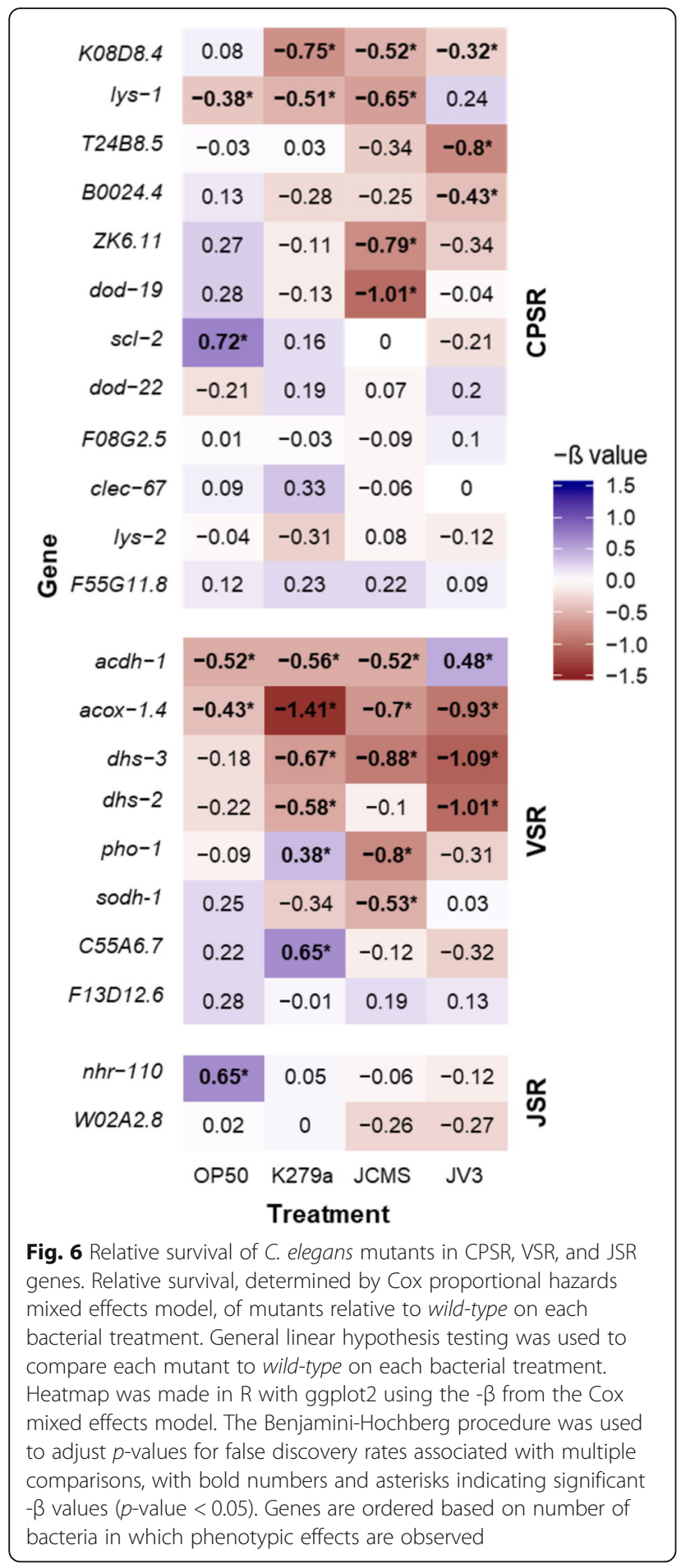

common sites of pathogen infection, whereas the nervous system has also been shown to play a role in pathogen recognition and immune response in $C$. elegans [40-43]. Therefore, expression of differentially expressed genes in response to $S$. maltophilia correlates with common tissues involved in innate immune response.

\section{Discussion}

Patterns of gene expression in response to different levels of S. maltophilia virulence

This study utilized a transcriptomic approach to identify genetic responses to $S$. maltophilia strains of differing pathogenicity. Our previous study that identified differentially expressed genes in response to E. coli OP50, S. maltophilia K279a, and S. maltophilia JCMS after $24 \mathrm{~h}$ found almost all genes were differentially expressed between nonpathogenic K279a and pathogenic JCMS, suggesting that the nematode response differs only to the presence of virulence. This study further examines these findings by adding an even more pathogenic strain, S. maltophilia JV3, to determine whether there are strain similarities and differences based on level of virulence. In this case, we identified differences in the nematode response between species ( $E$. coli and $S$. maltophilia) and levels of virulence (Figs. 2, and 3). However, the expression patterns between the nonpathogenic strains are more similar than those of the pathogenic strains (Fig. 2), suggesting that although there are differences between species, the majority of gene expression changes are based on the presence of virulence.

To determine the existence of a common pathogenic S. maltophilia response (CPSR), we identified 145 genes that were differentially expressed between all pathogenic and non-pathogenic comparisons (Fig. 3; Supplemental Table 3, Additional File 1). A majority of these genes $(89 \%)$ were upregulated in response to JCMS and JV3 (Supplemental Table 3, Additional File 1). This is consistent with previous transcriptomic patterns of genes differentially expressed in C. elegans upon pathogen exposure, in which a majority of genes were upregulated in response to a variety of bacterial and fungal pathogens $[23,24,44]$. However, another study that compared transcriptomic responses to a variety of bacterial pathogens found that a majority of genes were downregulated in response to Gram-negative bacteria and upregulated in response to Gram-positive bacteria [45]. Furthermore, our previous study of the response of $C$. elegans to $S$. maltophilia JCMS found a majority of genes were down-regulated [25]. However, that study used microarrays to detect expression differences induced at $24 \mathrm{~h}$ of exposure, whereas here we used RNA sequencing at $12 \mathrm{~h}$ of exposure. Therefore, this directionality of gene expression could be strain- or timespecific. In fact, 200 of the 225 (89\%) of the genes specifically differentially expressed in response to JV3 (VSR genes) are downregulated (Supplemental Table 5, Additional File 1). This supports the idea that directionality of gene expression in C. elegans may be strain specific, and different virulence mechanisms or host responses may play a role in these patterns. 
Table 3 Cox proportional hazard ratios for common S. maltophilia and strain-specific genes

\begin{tabular}{|c|c|c|c|c|c|c|c|}
\hline & \multirow[b]{2}{*}{ Nematode } & \multirow[b]{2}{*}{ Bacteria } & \multirow[b]{2}{*}{$\mathrm{N}$} & \multirow[b]{2}{*}{ M } & \multirow[b]{2}{*}{ SE } & \multicolumn{2}{|c|}{ Relative to wildtype } \\
\hline & & & & & & $\begin{array}{l}\text { Hazard Ratio } \\
\exp (\beta)\end{array}$ & $p$ value \\
\hline & wildtype & OP50 & 516 & 10.59 & 0.23 & NA & \\
\hline & & K279a & 615 & 10.87 & 0.19 & NA & \\
\hline & & JCMS & 608 & 5.44 & 0.07 & NA & \\
\hline & & JV3 & 580 & 2.53 & 0.03 & NA & \\
\hline \multirow[t]{40}{*}{ CPSR genes } & B0024.4 & OP50 & 26 & 10.50 & 0.78 & 0.88 & 0.698 \\
\hline & $(m h 82)$ & K279a & 58 & 8.66 & 0.55 & 1.32 & 0.146 \\
\hline & & JCMS & 90 & 4.74 & 0.16 & 1.28 & 0.14 \\
\hline & & JV3 & 58 & 2.06 & 0.07 & 1.54 & $.03^{*}$ \\
\hline & F08G2.5 & OP50 & 46 & 10.43 & 0.77 & 0.99 & 0.988 \\
\hline & $(m h 86)$ & K279a & 59 & 10.85 & 0.68 & 1.03 & 0.897 \\
\hline & & JCMS & 57 & 5.39 & 0.23 & 1.10 & 0.696 \\
\hline & & JV3 & 59 & 2.72 & 0.07 & 0.91 & 0.68 \\
\hline & ZK6.11 & OP50 & 46 & 12.24 & 0.74 & 0.76 & 0.194 \\
\hline & (ok3738) & K279a & 57 & 10.39 & 0.62 & 1.12 & 0.636 \\
\hline & & JCMS & 58 & 4.47 & 0.14 & 2.19 & $1.33 \mathrm{E}-6^{*}$ \\
\hline & & JV3 & 56 & 2.27 & 0.08 & 1.41 & 0.07 \\
\hline & T24B8.5 & OP50 & 58 & 10.69 & 0.60 & 1.04 & 0.897 \\
\hline & (ok3236) & K279a & 61 & 10.20 & 0.60 & 0.97 & 0.923 \\
\hline & & JCMS & 60 & 4.88 & 0.16 & 1.41 & 0.053 \\
\hline & & JV3 & 57 & 1.94 & 0.07 & 2.23 & $8.9 \mathrm{E}-7^{*}$ \\
\hline & dod-19 & OP50 & 56 & 11.84 & 0.64 & 0.76 & 0.14 \\
\hline & (ok2679) & K279a & 60 & 9.80 & 0.57 & 1.13 & 0.577 \\
\hline & & JCMS & 60 & 4.07 & 0.12 & 0.36 & 7.27E-11* \\
\hline & & JV3 & 58 & 2.36 & 0.10 & 1.04 & 0.873 \\
\hline & dod-22 & OP50 & 74 & 10.22 & 0.54 & 1.23 & 0.216 \\
\hline & (ok1918) & K279a & 90 & 13.23 & 0.41 & 0.83 & 0.216 \\
\hline & & JCMS & 89 & 5.87 & 0.18 & 1.07 & 0.702 \\
\hline & & JV3 & 89 & 0.28 & 0.08 & 0.82 & 0.194 \\
\hline & K08D8.4 & OP50 & 79 & 10.95 & 0.66 & 0.92 & 0.696 \\
\hline & $(m h 101)$ & K279a & 90 & 7.62 & 0.50 & 2.11 & 1.46E-8* \\
\hline & & JCMS & 87 & 4.82 & 0.17 & 1.68 & $.000154^{*}$ \\
\hline & & JV3 & 85 & 2.36 & 0.08 & 1.37 & $.038^{*}$ \\
\hline & lys-1 & OP50 & 82 & 10.09 & 0.42 & 1.46 & $.010^{*}$ \\
\hline & (ok2445) & K279a & 90 & 9.17 & 0.44 & 1.67 & $.000147^{*}$ \\
\hline & & JCMS & 88 & 4.76 & 0.14 & 1.92 & $1.33 \mathrm{E}-6^{*}$ \\
\hline & & JV3 & 86 & 2.77 & 0.08 & 0.78 & 0.129 \\
\hline & clec-67 & OP50 & 79 & 11.56 & 0.53 & 0.92 & 0.681 \\
\hline & (ok2770) & K279a & 55 & 13.20 & 0.68 & 0.71 & 0.077 \\
\hline & & JCMS & 57 & 5.46 & 0.24 & 1.06 & 0.8 \\
\hline & & JV3 & 53 & 2.67 & 0.10 & 1.00 & 0.988 \\
\hline & lys-2 & OP50 & 85 & 10.44 & 0.50 & 1.04 & 0.853 \\
\hline & (tm2398) & K279a & 58 & 10.21 & 0.54 & 1.36 & 0.077 \\
\hline & & JCMS & 58 & 5.71 & 0.19 & 0.92 & 0.698 \\
\hline & & JV3 & 55 & 2.59 & 0.08 & 1.13 & 0.606 \\
\hline
\end{tabular}


Table 3 Cox proportional hazard ratios for common S. maltophilia and strain-specific genes (Continued)

\begin{tabular}{|c|c|c|c|c|c|c|c|}
\hline & \multirow[b]{2}{*}{ Nematode } & \multirow[b]{2}{*}{ Bacteria } & \multirow[b]{2}{*}{ N } & \multirow[b]{2}{*}{ M } & \multirow[b]{2}{*}{ SE } & \multicolumn{2}{|c|}{ Relative to wildtype } \\
\hline & & & & & & $\begin{array}{l}\text { Hazard Ratio } \\
\exp (\beta)\end{array}$ & $p$ value \\
\hline & F55G11.8 & OP50 & 59 & 10.51 & 0.61 & 0.89 & 0.601 \\
\hline & $(g k 3130)$ & K279a & 58 & 11.55 & 0.51 & 0.79 & 0.216 \\
\hline & & JCMS & 60 & 5.65 & 0.19 & 0.80 & 0.217 \\
\hline & & $\mathrm{JV} 3$ & 57 & 2.44 & 0.10 & 0.92 & 0.698 \\
\hline & $s \mathrm{Cl}-2$ & OP50 & 51 & 15.43 & 0.73 & 0.49 & $2.97 \mathrm{E}-5^{*}$ \\
\hline & (tm2428) & K279a & 54 & 11.72 & 0.64 & 0.85 & 0.45 \\
\hline & & JCMS & 56 & 5.75 & 0.17 & 1.00 & 0.988 \\
\hline & & JV3 & 55 & 2.42 & 0.12 & 1.24 & 0.268 \\
\hline \multirow[t]{32}{*}{ VSR genes } & acdh-1 & OP50 & 50 & 7.12 & 0.77 & 1.68 & $.00688^{*}$ \\
\hline & (ok1489) & K279a & 59 & 8.53 & 0.49 & 1.74 & $.00178^{*}$ \\
\hline & & JCMS & 58 & 4.59 & 0.19 & 1.68 & $.00427^{*}$ \\
\hline & & $\mathrm{JV} 3$ & 58 & 2.68 & 0.11 & 0.62 & $.008^{*}$ \\
\hline & sodh-1 & OP50 & 53 & 10.55 & 0.64 & 1.09 & 0.698 \\
\hline & (ok2799) & K279a & 54 & 9.48 & 0.56 & 1.41 & 0.065 \\
\hline & & JCMS & 56 & 4.71 & 0.15 & 1.7 & $.00164^{*}$ \\
\hline & & JV3 & 55 & 2.5 & 0.11 & 0.97 & 0.897 \\
\hline & pho-1 & OP50 & 54 & 12.11 & 0.69 & 0.78 & 0.211 \\
\hline & (tm5302) & K279a & 55 & 12.47 & 0.74 & 0.68 & $.0405^{*}$ \\
\hline & & JCMS & 55 & 4.58 & 0.18 & 2.22 & $1.33 \mathrm{E}-6^{*}$ \\
\hline & & JV3 & 60 & 2.33 & 0.11 & 1.36 & 0.088 \\
\hline & C55A6.7 & OP50 & 57 & 11.91 & 0.69 & 0.80 & 0.234 \\
\hline & (tm6807) & K279a & 59 & 13.51 & 0.75 & 0.52 & $4.71 \mathrm{E}-5^{*}$ \\
\hline & & JCMS & 59 & 5.39 & 0.15 & 1.13 & 0.6 \\
\hline & & JV3 & 57 & 2.4 & 0.07 & 1.37 & 0.077 \\
\hline & acox-1.4 & OP50 & 58 & 9.91 & 0.42 & 1.53 & $.0137^{*}$ \\
\hline & (tm6415) & K279a & 60 & 6.2 & 0.33 & 4.10 & $<2 \mathrm{E}-16^{*}$ \\
\hline & & JCMS & 57 & 4.63 & 0.17 & 2.02 & $1.47 \mathrm{E}-5^{*}$ \\
\hline & & JV3 & 55 & 2.03 & 0.08 & 2.53 & $1.38 \mathrm{E}-8^{*}$ \\
\hline & dhs-3 & OP50 & 55 & 12 & 0.67 & 1.20 & 0.37 \\
\hline & (tm6151) & K279a & 58 & 9.16 & 0.65 & 1.95 & $4.45 \mathrm{E}-5^{*}$ \\
\hline & & JCMS & 55 & 4.87 & 0.15 & 2.41 & $4.04 \mathrm{E}-8^{*}$ \\
\hline & & $\mathrm{JV} 3$ & 55 & 2.04 & 0.08 & 2.97 & $2.31 \mathrm{E}-9^{*}$ \\
\hline & F13D12.6 & OP50 & 53 & 11.92 & 0.64 & 0.75 & 0.146 \\
\hline & (tm7051) & K279a & 58 & 10.98 & 0.51 & 1.01 & 0.216 \\
\hline & & JCMS & 56 & 6 & 0.15 & 0.82 & 0.32 \\
\hline & & JV3 & 57 & 2.59 & 0.12 & 0.88 & 0.566 \\
\hline & $d h s-2$ & OP50 & 27 & 9.3 & 0.80 & 1.24 & 0.474 \\
\hline & $(\operatorname{tm7516)}$ & K279a & 53 & 7.72 & 0.43 & 1.78 & $.00095^{*}$ \\
\hline & & JCMS & 53 & 4.91 & 0.20 & 1.11 & 0.681 \\
\hline & & JV3 & 53 & 1.64 & 0.09 & 2.74 & $2.35 \mathrm{E}-11^{*}$ \\
\hline \multirow[t]{4}{*}{ JSR genes } & nhr-110 & OP50 & 30 & 14.23 & 0.77 & 0.52 & $.00427^{*}$ \\
\hline & (gk987) & K279a & 58 & 9.69 & 0.57 & 0.95 & 0.853 \\
\hline & & JCMS & 58 & 5.16 & 0.13 & 1.06 & 0.8 \\
\hline & & $\mathrm{JV} 3$ & 57 & 2.29 & 0.07 & 1.13 & 0.619 \\
\hline
\end{tabular}


Table 3 Cox proportional hazard ratios for common S. maltophilia and strain-specific genes (Continued)

\begin{tabular}{|c|c|c|c|c|c|c|}
\hline \multirow[b]{2}{*}{ Nematode } & \multirow[b]{2}{*}{ Bacteria } & \multirow[b]{2}{*}{$\mathrm{N}$} & \multirow[b]{2}{*}{ M } & \multirow[b]{2}{*}{ SE } & \multicolumn{2}{|c|}{ Relative to wildtype } \\
\hline & & & & & $\begin{array}{l}\text { Hazard Ratio } \\
\exp (\beta)\end{array}$ & $p$ value \\
\hline W02A2.8 & OP50 & 59 & 11.27 & 0.56 & 0.98 & 0.929 \\
\hline$(m h 87)$ & K279a & 59 & 10.42 & 0.65 & 1.00 & 0.988 \\
\hline & JCMS & 59 & 5.22 & 0.17 & 1.29 & 0.15 \\
\hline & JV3 & 57 & 2.35 & 0.1 & 1.31 & 0.14 \\
\hline
\end{tabular}

Mean survival (M), standard error of the mean (SE), and sample size (N), are given for each nematode genotype and bacterial treatment combination. Wild-type statistics were determined from combining all wild-type data from all experiments. Hazard ratios (natural log $(\beta)$ ) indicate the treatment hazard divided by the hazard of wild-type (first column) across all experiments. The hazard is defined as the probability of a nematode dying at a given time. Hazard ratios and associated FDR adjusted p-values for each comparison were determined using Cox proportional hazards mixed effects model and general linear hypothesis tests and applying the Benjamini-Hochberg procedure to adjust for multiple comparisons in R. Asterisk indicate significant $\mathrm{p}$ values $(p<0.05)$

In comparison to VSR genes (225), there are very few genes (14) specifically differentially expressed in response to JCMS (JSR) (Supplemental Table 4, Additional File 1) and most of these genes were expressed at low levels. Overall, this suggests that at $12 \mathrm{~h}$ of exposure JCMS does not employ unique virulence mechanisms as it appears JV3 does and therefore does not lead to unique host responses.

\section{The CPSR and VSR genes identify functions that play different roles in the response to $S$. maltophilia}

We used GO enrichment and functional analysis of mutations to begin to understand functional roles of the CPSR genes. GO enrichment analysis identified processes involved in defense response, particularly response to Gram-negative bacteria, as well as a molecular function in carbohydrate binding and the cellular component of membrane raft (Table 1). Genes with the GO term "molecular function of carbohydrate binding" all belong to clec or lec family, which are structurally similar to carbohydrate binding proteins. Although not all nematode clec and lec genes encode molecules that bind carbohydrates, many are predicted to be secreted proteins that may act as immune effectors [34, 46]. Many clec genes are expressed in the $C$. elegans intestine and are differentially expressed in response to pathogens; a recent review determined that 237 of 283 clec genes are differentially expressed during pathogen infection [46]. We analyzed a mutation in only one clec gene, clec-67, and no differences were found in survival between the clec-67 mutant and wild-type. This might be explained by functional redundancy among the clec family proteins, as many of these proteins are structurally similar. In fact, there are nine other clec genes among the CPSR genes, three of which (clec-70, clec-83, and clec-85) are paralogous to clec-67, suggesting similar functional roles. Therefore, even though these genes may play a role in innate immune response, mutations in one clec gene alone may not result in an effect on survival upon pathogen-challenge. Future work to interfere with the functions of multiple clec genes at one time will shed light on this possibility.

Membrane rafts, or lipid rafts, are membrane domains that contain high concentrations of cholesterol and glycosphingolipids [47]. Membrane rafts also serve as sites of colocalization between membrane proteins and signaling pathway components, such as components of MAPK and insulin-like signaling pathways, both of which are known to play roles in innate immunity and defense in C. elegans [19-21, 47, 48]. In addition, many of the CPSR membrane raft genes also have biological processes associated with the innate immune response. To further support this, mutations in three paralogous membrane raft genes, B0024.4, ZK6.11, and dod-19, caused increased susceptibility to either JCMS or JV3. Mutations in two other genes, T24B8.5 and lys-1, were also shown to increase susceptibility to at least one $S$. maltophilia strain. T24B8.5 is regulated by the PMK-1 pathway [49], and lys-1 is regulated by the DBL-1 pathway [22], both well-known innate immune pathways. Therefore, it is possible that these membrane raft proteins are involved in innate immune signaling pathways, where increasing expression of the membrane raft proteins results in increased signaling and therefore increased expression of innate immune effector genes. Further analysis of these membrane raft genes and their potential role in innate immune signaling pathways could reveal novel gene functions that are important components in the response to S. maltophilia and potentially other pathogens.

On the other hand, GO enrichment analysis of VSR genes identified many processes and functions involved in metabolism (Table 2). Genes with GO terms "flavonoid glucuronidation", "flavonoid biosynthetic process", and "UPD-glycosyltransferase" consist almost entirely of glycosyltransferase family proteins, a large protein family in C. elegans, comprising 265 genes [50]. However, a direct linkage between 


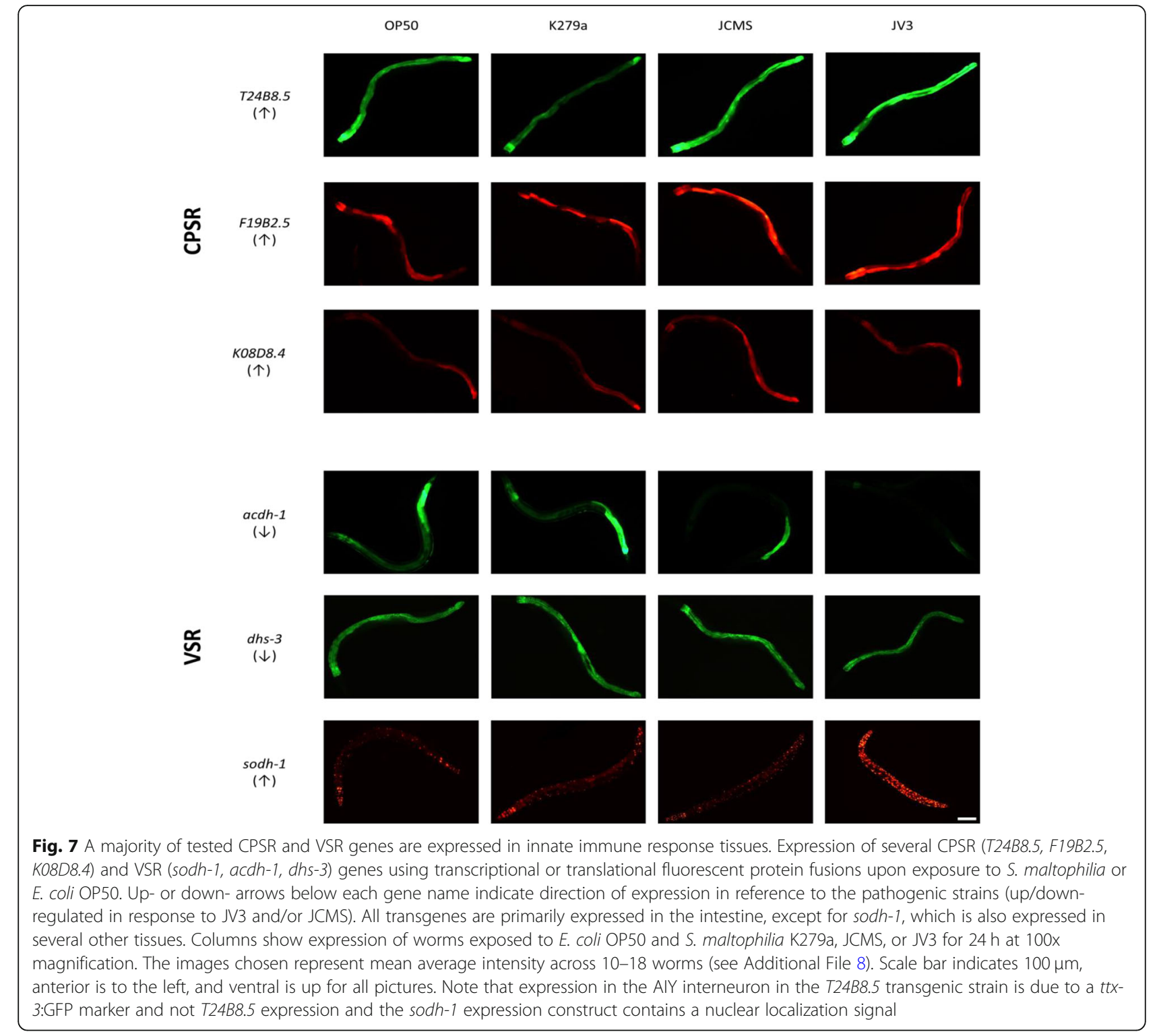

glycosyltransferases and innate immunity has not yet been observed. Genes with GO term "oxidation-reduction process" include many dehydrogenase and oxidase enzymes. Interestingly, mitochondria, the location of many dehydrogenases, have been shown to be involved in pathogen recognition [51]. Specifically, $\mathrm{FADH}_{2}$-dependent dehydrogenase activity in mice macrophages increases upon exposure to E. coli [52]. Furthermore, GO terms associated with metabolic processes have previously been found to be enriched among down-regulated genes in response to S. maltophilia and B. thuringiensis [25, 27]. One explanation might be that some pathogens, including S. maltophilia JV3, utilize mechanisms to interfere with metabolism in C. elegans, resulting in their own increased virulence. On the other hand, this downregulation may be a defense mechanism used by $C$. elegans to decrease metabolites needed for pathogen survival or pathogenesis. Five genes in this category, dhs-2, dhs-3, acdh-1, sodh-1, and acox-1.4, were chosen for functional analysis, and mutations in all had an effect on survival in response to at least one $S$. maltophilia strain. The involvement of VSR genes in general metabolism could explain their more general effect on survival (Fig. 6). However, further analyses of these genes in $C$. elegans are needed to fully understand their role in response to S. maltophilia JV3 and other pathogens. These analyses, along with JV3 genome sequence analysis, could provide insight into unique virulence mechanisms employed by JV3. 
In addition to strain-specific gene expression patterns, we also observe strain-specificity in our functional analyses (Fig. 6). For example, while mutations in the VSR gene $a c d h-1$ increase susceptibility to OP50, K279a, and JCMS, the same mutation causes a significant decrease in susceptibility to JV3. A similar pattern is observed with mutations in the CPSR gene lys- 1 that also cause increased susceptibility to OP50, K279a, and JCMS, but non-significantly decreased susceptibility to JV3. This further supports our hypothesis that JV3 utilizes unique mechanisms that result in strain-specific genetic responses in C. elegans. Therefore, further understanding the function of $l y s-1$ and $a c d h-1$ and their role in response to JV3 may uncover information about its virulence mechanisms.

\section{Host responses could exist along a continuum of response to increased pathogenicity}

Overall, data from the survival analyses do not support the simple hypothesis that CPSR genes are necessary for survival on JCMS and JV3, while JSR and VSR genes are necessary for survival on only JCMS or JV3, respectively, as mutations in a majority of genes did not affect survival of C. elegans in that strain-specific manner (Fig. 6; Table 3). However, it appears that overall, these genes do play a role in response to S. maltophilia, as 13 of 22 candidate CPSR, JSR, and VSR gene mutants display significant differences in survival upon exposure to at least one S. maltophilia strain. Furthermore, mutations in many of the genes we analyzed caused significant differences in survival upon exposure to K279a in addition to the pathogenic strains, JCMS and JV3 (Fig. 6; Table 3). Genome sequencing of S. maltophilia K279a has identified a variety of virulence factors [53], suggesting that it may in fact have pathogenic potential. Whereas wildtype $C$. elegans may be able to defend against K279a infection, mutations in innate immune and defense genes may cause $C$. elegans to become more susceptible to K279a. Therefore, identifying and functionally analyzing differentially expressed genes in C. elegans in response to all S. maltophilia strains could also uncover important genetic components of the response to S. maltophilia virulence.

Although RNA sequencing examines gene expression on a genome-wide scale, it only captures expression profiles at one point in time. Therefore, it is possible that the interaction between $C$. elegans and the different $S$. maltophilia strains is more nuanced than predicted by the simple hypothesis that genes differentially expressed at one point in time will affect nematode survival. For example, because JV3 is more virulent than JCMS and K279a, the JV3-exposed worms could be at a more advanced stage of pathogenesis than the JCMS and K279a-exposed worms at
$12 \mathrm{~h}$. Thus, in addition to any strain specific effects, differential responses at a single time point might also reflect different stages of pathogenesis. This idea is supported by our observation that among the upregulated CPSR genes, most were further upregulated in response to JV3 as compared to JCMS (Supplemental Table 3, Additional File 1). Survival analysis, unlike RNA-sequencing, can provide information about gene function across life history. The observation that mutations in many genes differentially expressed in response to specific $S$. maltophilia strains affect survival upon exposure to multiple strains of $S$. maltophilia suggests that these genes may be important for response to $S$. maltophilia more generally. That these mutations can cause varying effects on survival in response to different strains also indicates complexities of gene function within this general response. Transcriptional analysis of the $C$. elegans response to different strains across time of exposure could help determine the relative importance of time-specific and strain-specific responses.

\section{Conclusion}

Using transcriptomic data to understand and analyze responses to pathogens can provide insight into overall response patterns and pathogen virulence mechanisms. Utilizing both transcriptional and functional analyses, this study illustrates the previously supported idea that there are common signatures of pathogen infection in C. elegans, but also unique species and even strain specific responses. More specifically, we provide evidence to support that different strains of $S$. maltophilia of varying pathogenicity cause different transcriptomic signatures, and JV3 elicits a unique downregulation of metabolic genes in $C$. elegans that are involved in survival more generally. Therefore, to fully understand virulence of bacteria and pathogenesis in $C$. elegans for both clinical and environmental applications, a variety of bacterial species and strains need to be investigated. Because S. maltophilia is a human opportunistic pathogen, understanding virulence mechanisms of and host responses to a variety of $S$. maltophilia strains could lead to novel information about $S$. maltophilia infection. Finally, focusing on more natural host-pathogen interactions promises to provide a more realistic understanding of host responses.

\section{Methods}

Nematode and bacteria strains and growth

The following C. elegans strains were obtained from the CGC: RB1573 dod-22(ok1918), VC1749

F55G11.8(gk3130) ZK185.2(gk828), $\quad$ VC3059 ZK6.11(ok3738), VC2477 T24B8.5(ok3236), RB1893 lys1(ok2445), VC2249 dod-19(ok2679), RB2095 clec- 
67(ok2770), VC2176 nhr-110 (gk987), RB2114 sodh-1 (ok2799), VC1011 acdh-1(ok1489), LIU1 [dhs-3p::dhs-3:: GFP + unc-76(+)], AU78 [T24B8.5p::GFP:: unc-54-3' UTR + ttx-3p::GFP::unc-54-3' UTR], CF2124 [sodh-1p:: RFP (NLS) + rol-6(su1006)], VL717 [acdh-1p::GFP]. C. elegans strains containing the following alleles were obtained from the National BioResource Project (NBRP): lys-2(tm2398), scl-2(tm2428), dhs-3(tm6151), F13D12.6(tm7051), pho-1(tm5302), C55A6.7(tm6807), dhs-2(tm7516), acox-1.4(tm6415). All alleles were outcrossed 4 times and were screened by PCR after each outcross to obtain homozygous mutants. Forward and reverse primers used to test for each allele can be found on Wormbase. Each of the mutations used in this study have molecular lesions that indicate loss of gene function. Specific details of each mutation and its molecular lesion can be found on Wormbase. Bristol N2 strain was also obtained from the CGC and used as wild-type. All strains were maintained on nematode growth media (NGM) plates seeded with E. coli OP50 at $20^{\circ} \mathrm{C}$.

C. elegans strains containing the following expression constructs and alleles were generated as described below: mhEx284[F19B2.5p::F19B2.5::wrmScarlet::unc-543' UTR] and $m h E x 283[K 08 D 8.4 p:: K 08 D 8.4::$ wrmScarlet:: unc-54-3' UTR], F08G2.5(mh86), K08D8.4(mh76), B0024.4(mh82), W02A2.8(mh87).

Bacterial strains include E. coli OP50 from the CGC, Stenotrophomonas maltophilia JCMS isolated by our lab in association with nematodes from Konza Prairie near Manhattan, KS [12], Stenotrophomonas maltophilia K279a from R. Ryan (University College Cork), Stenotrophomonas maltophilia JV3 from J. Tiedje (Michigan State University).

All bacteria strains were frozen at $-80^{\circ} \mathrm{C}$ upon arrival and thawed frequently for experimentation. S. maltophilia strains are naturally Ampicillin resistant, thus were grown on Luria Broth (LB) agar containing $100 \mu \mathrm{g} / \mathrm{mL}$ Ampicillin to selectively isolate and maintain each strain while avoiding contamination. E. coli OP50 was grown on regular LB agar. Plates were incubated at $37^{\circ} \mathrm{C}$ overnight and kept at $4{ }^{\circ} \mathrm{C}$ thereafter. S. maltophilia strains were grown in liquid LB containing $100 \mu \mathrm{g} / \mathrm{mL}$ Ampicillin, and E. coli OP50 was grown in liquid LB and shaken overnight at $37^{\circ} \mathrm{C}$. Liquid cultures were then seeded onto NGM and grown at room temperature overnight before being used for experimentation.

\section{RNA isolation}

Wild-type nematodes were synchronized by bleaching, plated on E. coli OP50, and maintained at $20^{\circ} \mathrm{C}$. Synchronized larval stage 4 (L4) worms were washed several times in M9 buffer and transferred to treatment bacteria or E. coli OP50. Treatments included S. maltophilia strains K279a, JCMS, and JV3. After $12 \mathrm{~h}$ of exposure to treatment bacteria at $25^{\circ} \mathrm{C}$, worms were collected in M9 buffer and lysed in TRIzol ${ }^{\circ}$ (Life Technologies). $12 \mathrm{~h}$ of exposure to treatments was chosen because at this point bacterial accumulation in the intestine has begun [12], but almost all worms in each treatment were still alive. Only non-contaminated, un-starved populations were used for RNA extraction, and three biological replicates were collected for each treatment. Bulk RNA was extracted from these populations using PureLink RNA Mini Kit (Invitrogen), and DNase treated using OnColumn PureLink ${ }^{\bullet}$ DNase Treatment (Invitrogen) following the manufacturer's protocol. RNA quality was checked by determining $260 / 280$ and $260 / 230$ absorbance ratios using a NanoDrop ${ }^{\text {Tw }} 8000$ Spectrophotometer and observation of $18 \mathrm{~S}$ and $28 \mathrm{~S}$ rRNA bands using gel electrophoresis.

\section{RNA sequencing and analysis}

Extracted RNA was sent to the University of Kansas Center for Molecular Analysis of Disease Pathways Genome Sequencing core facility for library preparation and sequencing. Three biological replicates, consisting of pooled bulk nematode RNA, and two technical replicates of each biological replicate were sequenced for each treatment. Libraries were sequenced on Illumina HiSeq 2500 platform resulting in 100 base pair single-end reads. Sequence quality was assessed using FastQC.

Tophat 2, which uses the short-read mapping program Bowtie [54], was used to map reads to the $C$. elegans genome. Technical replicates were combined at this step. Transcriptome and genome versions WS235 were used as the reference. Minimum intron length was set to 15 base pairs $(-i 15)$ and the parameter for -no-novel-juncs was used. The remainder of settings were set to default. Cuffdiff, a program within Cufflinks, is used to compare expression of transcripts at the isoform-level between treatments, accounting for variability within biological replicates [55]. The parameter -multi-read-correct was used to account for reads mapping to multiple locations, with the remainder of settings set to default. Transcripts were considered significantly differentially expressed between treatments if the fold change $>2$ and the false discovery rate (FDR)-adjusted $p$-value $<0.05$. Heatmap analysis and comparison of differentially expressed genes between different conditions were performed in $\mathrm{R}$ (Vienna, Austria: $\mathrm{R}$ Foundation for Statistical Computing) using the heatmap.2 function in the package gplots. Fragments per kilobase per million (FPKM) values for each gene and treatment were $\log$ transformed, and gene expression values were centered and scaled to have mean zero and standard deviation one across the row. 


\section{Gene ontology enrichment analysis}

Differentially expressed genes of interest were queried for gene ontology (GO) term enrichment using DAVID Bioinformatics Resources 6.8 [29, 30] with the background set to the entire C. elegans gene list. Each gene is assigned one or more GO terms and categorized into Biological Process, Molecular Function, and Cellular Component. Gene sets of interest were analyzed using the functional annotation chart and the "ALL" database in DAVID for each GO category. Significant enrichment of GO terms was determined using a Fisher's exact test [30]; this test associates a $p$-value, or EASE score, to each GO term based on the number of genes associated with that term as compared to background [30]. Falsediscovery rate (FDR) correction of EASE scores, as implemented by DAVID, was used to control for multiple testing. GO terms were considered to be significantly enriched if FDR adjusted $p$-value $<0.05$.

\section{Mutant generation using CRISPR/Cas9}

CRISPR/Cas9 was used to generate mutations in W02A2.8, F08G2.5, K08D8.4, and B0024.4. Guide RNA (gRNA) sequences were chosen within the coding sequence of the gene of interest (GOI) using the CRISPRseek package in $\mathrm{R}$ to select guides with high efficacy, and CRISPR design (https://crispr.mit.edu) to identify possible off-target effects. Two to four gRNAs were identified and constructed for each GOI (Additional File 10). Double-stranded gRNA sequences consisted of 20 base pairs prior to the PAM site (NGG) plus overhanging base pairs on each end that overlapped with $B s a \mathrm{I}$-cut pRB1017 plasmid. This overlap allowed for proper ligation of the gRNA sequence into BsaI-cut pRB1017 [56].

A co-CRISPR method, described in Arribere et al., was used to facilitate detection of gene-editing events. Briefly, an injection mix of $50 \mathrm{ng} / \mu \mathrm{l}$ Peft:Cas 9 vector [57], 20-25 ng/ $\mu \mathrm{l}$ of $d p y-10$ gRNA [56], and $20-25 \mathrm{ng} / \mu \mathrm{l}$ of each target gRNA-carrying plasmid were injected into young adult worm gonads [58]. $F_{1}$ Dpy worms were then moved to new plates and allowed to lay eggs. DNA was then isolated from $F_{1}$ Dpy worms and amplified with primers targeting genomic sites flanking the gRNAs of the GOI (Additional File 10).

Gene-editing events were identified by differences in amplicon size as compared to wild-type, indicating an insertion or deletion in the gene. Worms containing mutant alleles were then sequenced to determine the molecular lesion and outcrossed twice to wild-type males to eliminate possible off-target mutations. None of the mutations we generated resulted in a visible phenotypic effect. The deletion in WO2A2.8 removes the start codon of the gene, whereas mutations in F08G2.5, K08D8.4, and B0024.4 result in a frameshift of the coding sequence. These molecular lesions suggest loss-of-function mutations. A summary of CRISPR/Cas9 generated alleles is shown in Additional File 4.

\section{Generation of expression construct strains}

NEBuilder HiFi DNA Assembly (New England BioLabs) was used to assemble the vector backbone (pPD95.75), promoter and gene of interest (GOI), and fluorescent tag (wrmScarlet). DNA vectors are assembled by ligating fragments with overlapping sequence using an endonuclease to create single-stranded overhangs within the overlap sequences and ligase to ligate the fragments together. In this case, the three fragments were generated via PCR using high fidelity Phusion DNA Polymerase (Thermo Fisher Scientific). Fragment 1, encoding the fluorescent protein wrmScarlet, was amplified from pSEM89_egl-23::SL2::wrmScarlet [59] using forward primer 5' - ATGGTCAGCAAGGGAGAGGCAG - 3' and reverse primer $5^{\prime}$ - TTACTTGTAGAGCTCGTCCATT CCTCC -3 '. Fragment 2, the plasmid pPD95.75, which contains GFP followed by the unc-54 3'UTR, was amplified using forward primer 5'- GACGAGCTCTACAA GTAACATTCGTAGAATTCCAACTGAGCG - $3^{\prime}$ and reverse primer $5^{\prime}-$ TTTTTCTACCGGTACCCT CCAAGGG - 3'. This generated a linearized vector backbone that included a majority of the plasmid, excluding the GFP coding sequence. Fragment 3, which contains the GOI driven by its endogenous promoter (either $2 \mathrm{~kb}$ upstream of the gene or to the nearest upstream gene) and differed for each gene, was amplified with the following primers: F19B2.5 driven by the F19B2.5 promoter (pF19B2.5::F19B2.5) forward primer 5' - GGAGGGTACCGGTAGAAAAATGATTATTTC CGGCTCGGG - 3' and reverse primer $5^{\prime}$ - CTCCCT TGCTGACCATCTGGCTGTCGTCGGCTC - 3', and K08D8.4 driven by the K08D8.4 promoter (pK08D8.4:: K08D8.4) forward primer 5' - GAGGGTACCGGTAGAAAAACACCCAAGGATTTGAAG - 3' and reverse primer 5'- CTCTCCCTTGCTGACCATGACCAGCA TAACAAAACC -3 '. The primers used to amplify the vector backbone and the promoter/GOI fragment contain the appropriate overlap sequence, resulting in circular assembly of the promoter and GOI fragment ligated to the wrmScarlet fragment ligated to the vector backbone. Fragments were then gel purified using PureLink ${ }^{\mathrm{m}}$ Quick Gel Extraction Kit (Invitrogen), followed by assembly and cloning using NEBuilder HiFi Assembly Master Mix and Cloning Kit following manufacturer's protocol.

Colonies containing possible positive constructs after cloning were tested by PCR to ensure the fragments were assembled correctly. DNA was extracted from confirmed correct colonies. Finally, $20-50 \mathrm{ng} / \mathrm{ul}$ of each 
construct along with $20 \mathrm{ng} / \mathrm{ul} d p y-10(+)$ plasmid were injected into Dpy worms. $\mathrm{F}_{2}$ wild-type worms were then screened for wrmScarlet expression, and 3 independent transgene-containing lines were obtained for each GOI, with one representative line chosen for further analysis.

\section{Gene expression analysis}

Nematodes containing extrachromosomal or integrated alleles for transcriptional or translational fluorescent protein fusions were moved to treatment bacteria (OP50, K279a, JCMS, or JV3) at the L4 stage. After 12 and $24 \mathrm{~h}$ of exposure to treatment bacteria, nematodes were anesthetized (10 $\mathrm{mM}$ sodium azide) for observation at 100x magnification using a Leica DM6 microscope equipped with epifluorescence and differential interference contrast (DIC) optics. To quantify expression, 1018 nematodes expressing each construct were imaged per treatment. We quantified expression within the intestine, the main immune organ of the worm, by marking the region posterior of the pharynx to the posterior intestine as a region of interest (ROI) and quantifying average fluorescent intensity (pixel sum within region / area) using LAX software (Leica). Mean average intensities were compared across treatments at each time point (12 and $24 \mathrm{~h}$ ) using a Tukey HSD multiple comparison test in R. Images were also taken of young adult worms containing expression constructs exposed to OP50 at 400x magnification to show localization of expression.

\section{C. elegans survival assays}

Treatment bacteria were cultured in liquid LB (with Ampicillin for S. maltophilia strains) overnight and $75 \mu \mathrm{l}$ of bacteria were plated onto NGM agar plates the day prior to use. Worms were bleached to synchronize and reared at $20^{\circ} \mathrm{C}$ on lawns of $E$. coli OP50. For survival assays, 10-12 L4 worms were transferred to each treatment plate, with three replicates of each treatment, and maintained at $25^{\circ} \mathrm{C}$. Worms were transferred to new plates every day until they stopped laying eggs to separate them from their progeny. Surviving worms were recorded each day and dead worms were removed from plates, as determined by lack of movement following prodding with a platinum wire pick. Plates that became contaminated or worms that crawled off the agar and died were removed from data analysis.

Statistical analyses were performed in $\mathrm{R}$ to determine differences between the independent variable nematode genotype, with the dependent variable being the probability of nematode death on a given day. Survival probability estimates over time were determined using the KaplanMeier formula using the survival package in R. The Cox proportional hazards mixed effects model was then used to compare the effects of nematode genotype using the coxme package in R. Wild-type worms were included in every round of experimentation, and during analysis, mutant nematode strains were compared to wild-type nematodes from all experimental rounds. The experimental rounds were treated as random variables and differences between rounds were accounted for within the model.

General linear hypothesis tested was performed using the multcomp package in $\mathrm{R}$ to compare mutant nematode strains to wild-type nematodes on each bacterial treatment. The Benjamini-Hochberg procedure was used to adjust $p$-values for false discovery rates associated with multiple comparisons, with adjusted $p$-values $<0.05$ considered to be significant. The relative survival heatmap was made in $\mathrm{R}$ with ggplot2 using the $-(\beta)$ value for each comparison to wild-type.

\section{Supplementary information}

Supplementary information accompanies this paper at https://doi.org/10. 1186/s12866-020-01771-1.

Additional file 1 Differentially expressed genes between treatment comparisons. List of all differentially expressed genes between comparisons. Genes were considered differentially expressed if foldchange $>2$ and FDR-adjusted $p$-value $<0.05$. Fragments per kilobase per million (FPKM) values are shown for each treatment. Additional sheets contain CPSR, VSR, and JSR genes with FPKM values and up/down-regulation in reference to the pathogenic strains (up/down-regulated in response to JV3 and/or JCMS). Asterisk indicates different direction of regulation depending on the comparison considered.

Additional file 2. GO analysis of upregulated CPSR and downregulated VSR genes. Gene ontology (GO) enrichment analysis was performed on the upregulated CPSR genes or the downregulated VSR genes using DAVID Bioinformatics Resources 6.8. GO analysis identifies terms relating to the biological process, molecular function, or cellular component that are significantly enriched among a list of genes. Indented terms indicate child terms, or subcategories, of the term listed above, with the parent term left-aligned. Note that the degree of indention of each term does not reflect absolute GO term level within each category. Count is the number of genes corresponding to each GO term. Percent is the count / total considered in analysis. FDR is the false discovery rate-corrected EASE enrichment score to account for multiple testing. Only terms with FDR < 0.05 and the most descriptive term for each unique gene list are shown.

Additional file 3. CPSR and VSR genes ordered based on gene network rank. WormNet v2 was queried with the 145 CPSR genes or 225 VSR genes. Connected genes are ordered based on WormNet score, which is based on the number of connections that gene has and the strength of the evidence for those connections (Score). C = number of connected CPSR genes to the listed gene. Up/down-regulated = direction of differential expression in response to pathogenic compared to nonpathogenic strains. Asterisk indicates different direction of regulation depending on the comparison considered.

Additional file 4 CRISPR/Cas9 generated alleles. Genes for which CRISPR/Cas9 alleles were generated are shown. Exons are indicated by green boxes and introns are indicated by black lines. Gene sizes are not to scale, but exon/intron size within genes is to scale. Relative location of the gRNAs is indicated by circles above the gene, and location of mutation is indicated in red (lines for deletions, triangles for insertions). All isoforms of K08D8.4 and W02A2.8 are shown. K08D8.4 mutations are predicted to result in loss of function of all isoforms; W02A2.8C may be expressed but is not differentially expressed between treatments. Mutation sequence and flanking sequence is shown on the right, with mutation sequence shown in red font (number in parentheses represents size of deletion)

Additional file 5 Mutations in CPSR genes result in a variety of survival patterns upon S. maltophilia exposure. Survivorship of wild-type 
nematodes and CPSR mutants on S. maltophilia JCMS, K279a, JV3, and E. coli OP50. Survival estimates were determined using Kaplan-Meier estimates generated in R. For these experiments, 10-12 worms were synchronized, picked onto each treatment bacterial lawn (3 plates per treatment/nematode combination) and the number of living worms was recorded daily. 2-3 replicates were completed for all bacterial and C. elegans strain combinations. Sample sizes, hazard ratios and $p$-values generated form Cox proportional hazards tests are shown in Table 3.

Additional file 6 Mutations in VSR genes result in a variety of survival patterns upon S. maltophilia exposure. Survivorship of wild-type nematodes and VSR mutants on S. maltophilia JCMS, K279a, JV3, and E. coli OP50. Survival estimates were determined using Kaplan-Meier estimates generated in R. For these experiments, 10-12 worms were synchronized, picked onto each treatment bacterial lawn (3 plates per treatment/nematode combination) and the number of living worms was recorded daily. 2-3 replicates were completed for all bacterial and C. elegans strain combinations. Sample sizes, hazard ratios and $p$-values generated form Cox proportional hazards tests are shown in Table 3.

Additional file $\mathbf{7}$ Mutations in JSR genes result in a variety of survival patterns upon S. maltophilia exposure. Survivorship of wild-type nematodes and JSR mutants on S. maltophilia JCMS, K279a, JV3, and E. coli OP50. Survival estimates were determined using Kaplan-Meier estimates generated in R. For these experiments, 10-12 worms were synchronized, picked onto each treatment bacterial lawn (3 plates per treatment/nematode combination) and the number of living worms was recorded daily. 2-3 replicates were completed for all bacterial and C. elegans strain combinations. Sample sizes, hazard ratios and $p$-values generated form Cox proportional hazards tests are shown in Table 3.

Additional file 8 Expression construct quantification at 12 and $24 \mathrm{~h}$. Expression levels of several CPSR (T24B8.5, F19B2.5, K08D8.4) and VSR (sodh-1, acdh-1, dhs-3) genes using transcriptional or translational fluorescent protein fusions upon exposure to S. maltophilia or E. coli OP50. L4 worms containing expression constructs were moved to $S$. maltophilia K279a, JCMS, JV3, or E. coli OP50. After 12 and $24 \mathrm{~h}$, images were taken of 10-18 worms for each treatment and expression construct, and average intensity was measured for each worm. Plots show mean average intensity and standard error for each time, treatment, and expression construct. Letters indicate significant differences across treatments for each time point (Tukey's HSD).

Additional file 9 Expression construct localization. Expression of several CPSR (T24B8.5, F19B2.5, K08D8.4) and VSR (sodh-1, acdh-1, dhs-3) genes using transcriptional or translational fluorescent protein fusions upon exposure to E. coli OP50 at 400x in young adult worms. T24B8.5, F19B2.5 and $d h s-3$ are only expressed in the intestine, so the anterior intestine is shown. K08D8.4, acdh-1 and sodh-1 are also expressed in the head, which is shown. Brackets indicate head and intestine region on each worm. Unclosed brackets signify that region extends out of frame. Scale bar indicates $20 \mu \mathrm{m}$, anterior is to the left, and ventral is up for all pictures. Note that expression in the AIY interneuron (arrow) in the T24B8.5 transgenic strain is due to a ttx-3:GFP marker and not T24B8.5 expression and the sodh-1 expression construct contains a nuclear localization signal.

Additional file $\mathbf{1 0}$ CRISPR/Cas9 target gene primers. Forward and reverse primers for each gRNA were annealed and ligated into pRB1017. gRNA sequence (20 bp prior to the PAM site) are underlined for each primer, non-underlined bases are included in the primer sequence for proper ligation into Bsal digested pRB1017. Primers flanking the gRNA target loci were used to amplify DNA from candidate mutant worms to detect insertions or deletions based on amplicon size. Odd numbers of flanking primers listed for any gene were tested in combinations (forward primer was tested with each reverse primers).

\section{Abbreviations}

FDR: False discovery rate; CPSR: Common pathogenic S. maltophilia response; JSR: JCMS-specific response; VSR: JV3-specific response; GO: Gene ontology; DAVID: Database for Annotation, Visualization, and Integrated Discovery; AUC: Area under curve; ROC: Receiver operating characteristic; FPKM: Fragment per kilobase per million; CRISPR: Clustered regularly interspersed short palindromic repeats

\section{Acknowledgements}

The authors thank Dr. Brad Olson, Dr. Wendy Reeves, and Dr. Chad Brassil for bioinformatics and statistical support, Sarah Hansen for experimental assistance, and Wormbase. We would also like to thank the National BioResource Project (NBRP) and the CGC, funded by NIH Office of Research Infrastructure Programs (P40 OD010440), for providing nematode strains.

\section{Authors' contributions}

Experimental design of the study was conducted by LR and MH. RNAsequencing was performed by LR and MH. Other experiments and data analyses were performed by LR. Manuscript was drafted by LR. LR and MH read, revised, and approved the final manuscript.

\section{Funding}

This work was supported by a sub award from NIH COBRE P20 GM103638 to E. Lundquist (University of Kansas), the Kansas State University Division of Biology and the University Nebraska-Lincoln School of Biological Sciences. These funding bodies had no role in the study design, data collection, analysis, interpretation or writing of the manuscript.

\section{Availability of data and materials}

The datasets generated and/or analyzed during the current study are available in the NCBI Sequence Read Archive (SRA) repository under BioProject ID PRJNA561173.

\section{Ethics approval and consent to participate} Not applicable.

\section{Consent for publication}

Not applicable.

\section{Competing interests}

The authors declare that they have no competing interests.

Received: 16 August 2019 Accepted: 29 March 2020

Published online: 19 June 2020

\section{References}

1. Brooke JS. Stenotrophomonas maltophilia: an emerging global opportunistic pathogen. Clin Microbiol Rev. 2012;25:2-41.

2. Elting LS, Bodey GP. Septicemia due to Xanthomonas species and nonaeruginosa Pseudomonas species: increasing incidence of catheter-related infections. Medicine (Baltimore). 1990;69:296-306.

3. Jägevall S, Rabe L, Pedersen K. Abundance and diversity of biofilms in natural and artificial aquifers of the Äspö hard rock laboratory, Sweden. Microb Ecol. 2011:61:410-22.

4. Chang Y-T, Lin C-Y, Chen Y-H, Hsueh P-R. Update on infections caused by Stenotrophomonas maltophilia with particular attention to resistance mechanisms and therapeutic options. Front Microbiol. 2015;6:1-20.

5. Adamek M, Linke B, Schwartz T. Virulence genes in clinical and environmental Stenotrophomas maltophilia isolates: a genome sequencing and gene expression approach. Microb Pathog. 2014;67-68:20-30.

6. Adamek M, Overhage J, Bathe S, Winter J, Fischer R, Adamek M. Genotyping of environmental and clinical Stenotrophomonas maltophilia isolates and their pathogenic potential. PLoS One. 2011;6:e27615.

7. Berg G, Roskot N, Smalla K. Genotypic and phenotypic relationships between clinical and environmental isolates of Stenotrophomonas maltophilia. J Clin Microbiol. 1999;37:3594-600.

8. Ferrer-Navarro M, Planell R, Yero D, Mongiardini E, Torrent G, Huedo P, Martinez P, Roher N, Mackenzie S, Gibert I, et al. Abundance of the quorumsensing factor Ax21 in four strains of Stenotrophomonas maltophilia correlates with mortality rate in a new Zebrafish model of infection. PLoS One. 2013;8(6):e67207

9. Lira F, Berg G, Martínez JL. Double-face meets the bacterial world: the opportunistic pathogen Stenotrophomonas maltophilia. Front Microbiol. 2017:8:2190

10. Rocco F, De Gregorio E, Colonna B, Di Nocera PP. Stenotrophomonas maltophilia genomes: a start-up comparison. Int J Med Microbiol. 2009;299: 535-46. 
11. Kaiser S, Biehler K, Jonas D. A Stenotrophomonas maltophilia multilocus sequence typing scheme for inferring population structure. J Bacteriol. 2009; 191:2934-43.

12. White CV, Darby BJ, Bredden RJ, Herman MA. A Stenotrophomonas maltophilia strain evades a major Caenorhabditis elegans defense pathway. Infect Immun. 2016:84:524-36.

13. Berg M, Stenuit B, Ho J, Wang A, Parke C, Knight M, Alvarez-cohen L, Shapira M. Assembly of the Caenorhabditis elegans gut microbiota from diverse soil microbial environments. ISME J. 2016;10:1998-2009.

14. Dirksen P, Marsh SA, Braker I, Heitland N, Wagner S, Nakad R, Mader S, Petersen C, Kowallik V, Rosenstiel $P$, et al. The native microbiome of the nematode Caenorhabditis elegans: gateway to a new host-microbiome model. BMC Biol. 2016;14:38.

15. Zhang F, Berg M, Dierking K, Félix M-A, Shapira M, Samuel BS, Schulenburg $H$. Caenorhabditis elegans as a model for microbiome research. Front Microbiol. 2017:8:1-10

16. Garigan D, Hsu A-L, Fraser AG, Kamath RS, Ahringer J, Kenyon C. Genetic analysis of tissue aging in Caenorhabditis elegans: a role for heat-shock factor and bacterial proliferation. Genetics. 2002;161:1101-12.

17. McGhee JD, Sleumer MC, Bilenky M, Wong K, McKay SJ, Goszczynski B, Tian $\mathrm{H}$, Krich ND, Khattra J, Holt RA, et al. The ELT-2 GATA-factor and the global regulation of transcription in the C. elegans intestine. Dev Biol. 2007;302: $627-45$.

18. Samuel BS, Rowedder H, Braendle C, Félix M-A, Ruvkun G. Caenorhabditis elegans responses to bacteria from its natural habitats. Proc Natl Acad Sci. 2016;113:E3941-9.

19. Aballay A, Drenkard E, Hilbun LR, Ausubel FM. Caenorhabditis elegans innate immune response triggered by Salmonella enterica requires intact LPS and is mediated by a MAPK signaling pathway. Curr Biol. 2003;13:47-52.

20. Kim DH, Feinbaum $R$, Alloing G, Emerson FE, Garsin DA, Inoue H, TanakaHino M, Hisamoto N, Matsumoto K, Tan M-W, et al. A conserved p38 MAP kinase pathway in Caenorhabditis elegans innate immunity. Science. 2002; 297:623-6.

21. Sifri CD, Begun J, Ausubel FM, Calderwood SB. Caenorhabditis elegans a71s a model host for Staphylococcus aureus pathogenesis. Infect Immun. 2003 71(4):2208-17.

22. Alper S, Mcbride SJ, Lackford B, Freedman JH, Schwartz DA. Specificity and complexity of the Caenorhabditis elegans innate immune response. Mol Cell Biol. 2007;27:5544-53.

23. Troemel ER, Chu SW, Reinke V, Lee SS, Ausubel FM, Kim DH. p38 MAPK regulates expression of immune response genes and contributes to longevity in C. elegans. PLoS Genet. 2006;2:e183.

24. Engelmann I, Griffon A, Tichit L, Montanana-Sanchis F, Wang G, Reinke V Waterston RH, Hillier LW, Ewbank JJ. A comprehensive analysis of gene expression changes provoked by bacterial and fungal infection in C. elegans. PLOS ONE. 2011;6:e19055

25. White CV, Herman MA. Transcriptomic, functional, and network analyses reveal novel genes involved in the interaction between Caenorhabditis elegans and Stenotrophomonas maltophilia. Front Cell Infect Microbiol. 2018; $8: 266$

26. Irazoqui JE, Troemel ER, Feinbaum RL, Luhachack LG, Cezairliyan BO, Ausubel FM. Distinct pathogenesis and host responses during infection of C. elegans by P. aeruginosa and S. aureus. PLoS Pathogens. 2010;6:e1000982.

27. Yang W, Dierking K, Esser D, Tholey A, Leippe M, Rosenstiel P, Schulenburg $\mathrm{H}$. Overlapping and unique signatures in the proteomic and transcriptomic responses of the nematode Caenorhabditis elegans toward pathogenic Bacillus thuringiensis. Dev Comp Immunol. 2015;51:1-9.

28. Cabreiro F, Gems D. Worms need microbes too: microbiota, health and aging in Caenorhabditis elegans. EMBO Mol Med. 2013;5:1300-10.

29. Huang DW, Sherman BT, Lempicki RA. Bioinformatics enrichment tools: paths toward the comprehensive functional analysis of large gene lists. Nucleic Acids Res. 2009;37:1-13

30. Huang DW, Sherman BT, Lempicki RA. Systematic and integrative analysis of large gene lists using DAVID bioinformatics resources. Nat Protoc. 2009;4: 44-57.

31. Lee I, Lehner B, Vavouri T, Shin J, Fraser AG, Marcotte EM. Predicting genetic modifier loci using functional gene networks. Genome Res. 2010;20:114353.

32. Lee I, Lehner B, Crombie C, Wong W, Fraser AG, Marcotte EM. A single gene network acurately predicts phenotypic effects of gene perturbation in Caenorhabditis elegans. Nat Genet. 2008:40:181-8.
33. Özgür A, Vu T, Erkan G, Radev DR. Identifying gene-disease associations using centrality on a literature mined gene-interaction network Bioinformatics. 2008;24:277-85.

34. Dierking $\mathrm{K}$, Yang W, Schulenburg $\mathrm{H}$. Antimicrobial effectors in the nematode Caenorhabditis elegans: an outgroup to the Arthropoda. Philos Trans R Soc B. 2016:371:20150299.

35. Mallo GV, Kurz CL, Couillault C, Pujol N, Granjeaud S, Kohara Y, Ewbank JJ. Inducible antibacterial defense system in C. elegans. Curr Biol. 2002;12:1209-14.

36. Chikina MD, Huttenhower C, Murphy CT, Troyanskaya OG. Global prediction of tissue-specific gene expression and context-dependent gene networks in Caenorhabditis elegans. PLoS Comput Biol. 2009;5(6):e1000417.

37. Fukushige T, Goszczynski B, Yan J, McGhee JD. Transcriptional control and patterning of the pho-1 gene, an essential acid phosphatase expressed in the C. elegans intestine. Dev Biol. 2005;279(2):446-61.

38. Nykamp K, Lee MH, Kimble J. C. elegans La-related protein, LARP-1, localizes to germline $P$ bodies and attenuates Ras-MAPK signaling during oogenesis. RNA. 2008;14(7):1378-89.

39. Steinbaugh MJ, Narasimhan SD, Robida-Stubbs S, Moronetti Mazzeo LE, Dreyfuss JM, Hourihan JM, Raghavan P, Operana TN, Esmaillie R, Blackwell TK. Lipid-mediated regulation of SKN-1/Nrf in response to germ cell absence. Elife. 2015:4:e07836.

40. Kawli T, Tan M-W. Neuroendocrine signals modulate the innate immunity of Caenorhabditis elegans through insulin signaling. Nat Immunol. 2008:9:1415-24.

41. Shtonda BB, Avery L. Dietary choice behavior in Caenorhabditis elegans. J Exp Biol. 2006;209:89-102.

42. Styer KL, Singh V, Macosko E, Steele SE, Bargmann Cl, Aballay A. Innate immunity in Caenorhabditis elegans is regulated by neurons expressing NPR1/GPCR. Science. 2008:322:460-5.

43. Zhang Y. Neuronal mechanisms of Caenorhabditis elegans and pathogenic bacteria interactions. Curr Opin Microbiol. 2008;11:257-61.

44. Boehnisch C, Wong D, Habig M, Isermann K, Michiels NK, Roeder T, May RC, Schulenburg H. Protist-type lysozymes of the nematode Caenorhabditis elegans contribute to resistance against pathogenic Bacillus thuringiensis. PLoS One. 2011:6:e24619.

45. Sinha A, Rae R, latsenko I, Sommer RJ. System wide analysis of the evolution of innate immunity in the nematode model species Caenorhabditis elegans and Pristionchus pacificus. PLoS One. 2012;7:e44255.

46. Pees B, Yang W, Zárate-potes A, Schulenburg H, Dierking K. High innate immune specificity through diversified C-type lectin-like domain proteins in invertebrates. J Innate Immun. 2016:8:129-42.

47. Pike LJ. Lipid rafts: bringing order to chaos. J Lipid Res. 2003;44:655-67.

48. Murphy CT, Lee S-J, Kenyon C. Tissue entrainment by feedback regulation of insulin gene expression in the endoderm of Caenorhabditis elegans. Proc Natl Acad Sci. 2007;104:19046-50

49. Pagano DJ, Kingston ER, Kim DH. Tissue expression pattern of PMK-2 p38 MAPK is established by the miR-58 family in C. elegans. PLoS Genet. 2015; 11(2):e1004997.

50. Yonekura-Sakakibara K, Hanada K. An evolutionary view of functional diversity in family 1 glycosyltransferases. Plant J. 2011;66:182-93.

51. Sancho D, Enamorado M, Garaude J. Innate immune function of mitochondrial metabolism. Front Immunol. 2017:8:1-9.

52. Garaude J, Acín-Pérez R, Martínez-Cano S, Enamorado M, Ugolini M, NistalVillán E, Hervás-Stubbs S, Pelegrín P, Sander LE, Enríquez JA, et al. Mitochondrial respiratory-chain adaptations in macrophages contribute to antibacterial host defense. Nat Immunol. 2016;17:1037-45.

53. Crossman LC, Gould VC, Dow JM, Vernikos GS, Okazaki A, Sebaihia M, Saunders D, Arrowsmith C, Carver T, Peters N, et al. The complete genome, comparative and functional analysis of Stenotrophomonas maltophilia reveals an organism heavily shielded. Genome Biol. 2008;9:R74.1-R74.13.

54. Kim D, Pertea G, Trapnell C, Pimentel H, Kelley R, Salzberg SL. TopHat2: accurate alignment of transcriptomes in the presence of insertions, deletions and gene fusions. Genome Biol. 2013;14:R36.

55. Trapnell C, Hendrickson DG, Sauvageau M, Goff L, Rinn JL, Pachter L. Differential analysis of gene regulation at transcript resolution with RNA-seq Nat Biotechnol. 2013;31:46-53.

56. Arribere JA, Bell RT, Fu BXH, Artiles KL, Hartman PS, Fire AZ. Efficient markerfree recovery of custom genetic modifications with CRISPR/Cas9 in Caenorhabditis elegans. Genetics. 2014:198:837-46.

57. Friedland AE, Tzur YB, Esvelt KM, Colaiacovo MP, Church GM, Calarco JA Heritable genome editing in C. elegans via a CRISPR-Cas9 system. Nat Methods. 2013;10:741-3. 
58. Evans T. Transformation and microinjection. WormBook; 2006

59. Mouridi SE, Lecroisey C, Tardy P, Mercier M, Leclerca-Blondel A, Zariohi N, Boulin T. Reliable CRISPR/Cas9 genome engineering in Caenorhabditis elegans using a single efficient sgRNA and an easily recognizable phenotype. G3. 2017;7:1429-37.

\section{Publisher's Note}

Springer Nature remains neutral with regard to jurisdictional claims in published maps and institutional affiliations.

Ready to submit your research? Choose BMC and benefit from:

- fast, convenient online submission

- thorough peer review by experienced researchers in your field

- rapid publication on acceptance

- support for research data, including large and complex data types

- gold Open Access which fosters wider collaboration and increased citations

- maximum visibility for your research: over $100 \mathrm{M}$ website views per year

At $B M C$, research is always in progress.

Learn more biomedcentral.com/submissions 